\title{
MODELING SHALLOW GRAVITY-DRIVEN SOLID-FLUID MIXTURES OVER ARBITRARY TOPOGRAPHY*
}

\author{
IOANA LUCA ${ }^{\dagger}$, YIH-CHIN TAI ${ }^{\ddagger}$, AND CHIH-YU KUO $§$
}

\begin{abstract}
The purpose of this paper is to derive modeling equations for debris flows on real terrain. Thus, we use curvilinear coordinates adapted to the topography as introduced, e.g., by Bouchut and Westdickenberg [F. Bouchut and M. Westdickenberg, Commun. Math. Sci., 2(3), 359389, 2004], and develop depth-averaged models of gravity-driven saturated mixtures of solid grains and pore fluid on an arbitrary rigid basal surface. First, by only specifying the interaction force and ordering approximations in terms of an aspect ratio between a typical length perpendicular to the topography, and a typical length parallel to the topography, we derive the governing equations for the shallow flow of a binary mixture, driven by gravitational force. In doing so, the non-uniformity through the avalanche depth of the constituent velocities and of the solid volume fraction is accounted for by coefficients of Boussinesq type. Then, the material behaviour peculiarities of both constituents properly enter the theory. One constituent is a granular solid. For its stresses we propose three models, one of them of Mohr-Coulomb type. The other constituent is a Newtonian/non-Newtonian fluid with small viscosity, obeying a viscous bottom friction condition. The final governing equations for the shallow flow of the mixture, incorporating the constitutive assumptions, are deduced, and the limiting equilibrium is then investigated.
\end{abstract}

Key words. Debris flow, solid-fluid mixture, curvilinear coordinates adapted to the topography, shallow avalanche equations, Mohr-Coulomb granular material.

AMS subject classifications. 76A20, 76D27, 76N20, 86A04.

\section{Introduction}

Geophysical flows across three-dimensional terrain include debris flows, which are collections of geological materials (e.g., sand, rocks) mixed with water, transporting trees, cars, pieces of destroyed houses, etc. The damages that debris flows produce are huge, hence the considerable interest in their prediction and evolution once initiated.

Three processes characterize a debris flow: initiation, movement and deposition. Due to their complexity, to this date there is no theoretical approach which could account for all these processes. This paper refers to the movement of the avalanching body, more specifically, to the derivation of the equations describing the motion of a shallow mass of debris on arbitrary rigid topography. For an extensive literature on modeling equations of avalanching flows the reader may consult Pudasaini and Hutter [22] and Harbitz [6]. Here we restrict the introductory survey to works closely related to the topic of the paper.

The efforts in deducing modeling equations for the flowing material layer concentrate on (i) the rheology of the avalanching mass and, more recently, on (ii) the account of the influences due to the geometry of the basal surface. Related to (i), as a real progress in comparison to earlier works, we mention the depth-integrated granular model of Mohr-Coulomb type developed by Savage and Hutter [24]. Very

*Received on July 24, 2008; accepted (in revised version): October 23, 2008. Communicated by François Bouchut.

${ }^{\dagger}$ Division of Mechanics, Research Center for Applied Sciences, Academia Sinica, Nangang, Taipei 11529, Taiwan; on leave from Department of Mathematics II, University Politehnica of Bucharest, Splaiul Independentei 313, 79590 Bucharest, Romania (iolucaro@yahoo.com).

${ }^{\ddagger}$ Department of Civil Engineering, National Chi Nan University, No. 1, University Road, Puli, Nantou 545, Taiwan (yctai@ncnu.edu.tw).

$\S$ Division of Mechanics, Research Center for Applied Sciences, Academia Sinica, Nangang, Taipei 11529, Taiwan (cykuo06@gate.sinica.edu.tw). 
close to this proposal are the models by Iverson [8], McDougall and Hungr [14], [15]. To (ii) we refer in more detail, since the main contribution of the present paper is the description of the moving mass on arbitrary topography. Thus, related to (ii), we mention Pudasaini et al. [19]-[21] for designing an orthogonal coordinate system suitable for a channel-type form of the bed surface, and Iverson and Denlinger [9], Pitman and Le [18] and Pelanti, Bouchut and Mangeney [17] for depth-averaged equations corresponding to topographic terrains with small slope variations.

However, the first general formulation of the modeling equations in coordinates following the real topography is due to Bouchut and Westdickenberg [1]. Unlike many works devoted to avalanche modeling, within this approach the avalanche depth is measured along the normal direction to the bed surface, and not along the vertical direction, which would distort the shallowness assumption in steep topographies. However, the rheological properties are so weak that the flowing material is an inviscid fluid, which is not a model that can describe, e.g., a deposition process. Independently, De Toni and Scotton [4] used essentially the same coordinate system as did Bouchut and Westdickenberg [1] to derive modeling equations for an avalanche mass of SavageHutter type, but they measured the avalanche depth vertically. By using the powerful matrix decomposition of field quantities and relations, as introduced in Bouchut and Westdickenberg [1], Luca, Tai and Kuo [10] reconsidered the formulation of modeling equations for the avalanche mass (inviscid fluid and Newtonian fluid) with the aid of the depth-averaging procedure. The approach could be easily extended in Luca et al. [11] to account for any rheologic behaviour, and to derive a hierarchy of depthintegrated avalanche models in terms of the scaling of the stress components; besides, a topography-adjusted version of the Savage-Hutter model fitting into this hierarchy has been proposed. We also mention Bouchut et al. [2] and Tai and Kuo [28] for including the erosion/deposition process into a framework which uses the topography following coordinates of Bouchut and Westdickenberg [1].

Most of the references cited above concern single-phase dry granular avalanches, which are clearly a rough approximation when dealing with debris flows. More realistic descriptions of these flows, that is as solid-fluid mixtures, are given by Iverson [8], Iverson and Denlinger [9], Pudasaini, Hutter and Wang [20], Pitman and Le [18], and recently by Pelanti, Bouchut and Mangeney [17]. Iverson [8] and Iverson and Denlinger [9] use the mass balance and momentum balance of the (saturated) mixture as a whole, with the fluid velocity estimated as being equal to the solid velocity, and constant solid volume fraction. The paper by Pudasaini, Hutter and Wang [20] is developed in the same spirit. On the other hand, Pitman and Le [18] derived model equations for a binary mixture, in which each constituent moves according to its own mass balance and momentum balance equations. As a consequence, the interaction force between constituents (the effect of which is annihilated in the models of Iverson type by adding the momentum balance equations of both constituents) plays an important role in the derivation of the constituent pressures. A slightly but substantially different version of Pitman and Le [18] is proposed in Pelanti, Bouchut and Mangenay [17], where, unlike Pitman and Le [18] (who depth-average the momentum balance equation corresponding to the fluid constituent after dividing by the fluid volume fraction), the momentum balance equation corresponding to the fluid constituent is depth-averaged in its original form, which is more reasonable if the analogy with the turbulence theory is invoked.

The approaches dealing with mixture models are restricted to topography with small slope variations of the bed surface. It is the purpose of the present paper to 
deal with a mixture on arbitrary topography. Thus, we extend the considerations in Luca et al. [11] to a two-phase flow. One constituent is a Newtonian/non-Newtonian fluid with small viscosity (so small that the stresses in the bulk fluid approximately reduce to the pore pressure), and experiencing bottom viscous friction. The other constituent is a granular material experiencing bottom Coulomb friction, and of which the stresses in the bulk material are given by (1) a spherical tensor, (2) a topographyadapted version of the Iverson-Denlinger model [9], or (3) the topography-adapted version of the Savage-Hutter model as introduced in Luca et al. [11]. Thus, three mathematical models for debris flows are developed. The interaction force, different from that in Pitman and Le [18], is carried over from Schneider and Hutter [25], where it is thermodynamically justified, but here it is postulated in an ad-hoc manner. An interesting consequence of this assumption is that both constituent pressures are essentially given by the same formulas as for the single-phase material in Luca et al. [11].

The content of the paper is structured as follows. Section 2 briefly introduces the geometric properties of the basal surface and the topography-based curvilinear coordinates as in Bouchut and Westdickenberg [1]. Section 3 formulates the intrinsic model equations for a binary saturated mixture, in a region which is bounded below by the topographic surface and above by the free surface, which, as in Pitman and Le [18], is considered a material surface for each constituent. A formal introduction of the constituent pressures is given. Later, these pressures are identified as the pore fluid pressure and the solid stress component acting perpendicular to a surface element parallel to the bed surface. Section 4 derives the non-dimensional model equations in the curvilinear coordinates described in section 2 . In section 5 the depthaveraged mass and tangential momentum balance equations corresponding to each constituent are deduced, and the basic equations which are next dealt with are clearly stated. Section 6 introduces the scaling approximations due to the shallowness of the flowing material layer. The non-uniformity along the avalanche depth of the tangential velocities and solid volume fraction are accounted for by coefficients of Boussinesq type. In section 7 the interaction force is postulated, and the emerging modeling equations, deduced in the Appendix without specifying the rheological properties of the constituents, are presented. Section 8 specifies the material peculiarities. Thus, three depth-integrated avalanche models for debris flows are proposed. In section 9 we take up the problem of finding the conditions satisfied by the solid volume fraction and avalanche depth when the avalanche mass reaches the equilibrium. Finally, section 10 is devoted to conclusions. Numerical investigations based on the derived model equations are left for future work.

In this paper we use the following notations. The $2 \times 2$ matrices are denoted by capital upright boldface letters, e.g., A, and the 2-column matrices are denoted by small upright boldface letters, e.g., a. A similar notation, but with slanted letters, is used for vectors and tensors, e.g., $\boldsymbol{a}, \boldsymbol{A}$. The dyadic product of two column matrices $\mathbf{a}$ and $\mathbf{b}$ is $\mathbf{a} \otimes \mathbf{b} \equiv \mathbf{a} \mathbf{b}^{T}$, where the superscript $T$ stands for the transpose of a matrix; the symbol $\otimes$ also denotes the tensor product of two vectors. The inner product of the 2-column matrices $\mathbf{a}$ and $\mathbf{b}$ is $\mathbf{a} \cdot \mathbf{b} \equiv \operatorname{tr}\left(\mathbf{a b}^{T}\right)$, where tr denotes the trace operator, and the inner product of the squared matrices $\mathbf{A}, \mathbf{B}$ is defined as $\mathbf{A} \cdot \mathbf{B} \equiv \operatorname{tr}\left(\mathbf{A B}^{T}\right)$. Finally, the Greek indices have the values 1, 2, the Latin indices range from 1 to 3, and summation over repeated indices is understood.

\section{Curvilinear coordinates adapted to the topography}

In this section we shall briefly present the curvilinear coordinates introduced by 
Bouchut and Westdickenberg [1], see also De Toni and Scotton [4], as a tool to derive modeling equations for the avalanche mass flowing on arbitrary topography. In doing so we follow the exposure from Luca et al. [11].

Thus, we suppose the mathematical model of the basal topography to be a regular surface $\mathcal{S}$ in the three-dimensional Euclidean point space $\mathcal{E}$, of which the translation vector space is denoted by $\mathcal{V}$. Assume that, with respect to an orthogonal Cartesian coordinate system $O x_{1} x_{2} x_{3}$, in which $O x_{3}$ is (physically) the vertical direction, $\mathcal{S}$ has the representation

$$
x_{1}=x_{1}\left(\xi^{1}, \xi^{2}\right), \quad x_{2}=x_{2}\left(\xi^{1}, \xi^{2}\right), \quad x_{3}=b\left(x_{1}\left(\xi^{1}, \xi^{2}\right), x_{2}\left(\xi^{1}, \xi^{2}\right)\right),
$$

which is considered of class $C^{2}$ on some open subset $\Delta_{0}$ of $\mathbb{R}^{2}$, and such that the determinant of the matrix

$$
\mathbf{F} \equiv\left(\frac{\partial x_{i}}{\partial \xi^{\alpha}}\right)_{i, \alpha \in\{1,2\}}
$$

is positive (det $\mathbf{F}>0$ ). Then, if $\boldsymbol{\rho} \in \mathcal{V}$ is the position vector of a point on $\mathcal{S}$, the vectors

$$
\boldsymbol{\tau}_{\alpha} \equiv \frac{\partial \boldsymbol{\rho}}{\partial \xi^{\alpha}}, \quad \alpha \in\{1,2\},
$$

define the natural basis of the tangent space to $\mathcal{S}$ at that point; moreover, a unit normal vector field to $\mathcal{S}$ is

$$
\boldsymbol{n} \equiv \frac{\boldsymbol{\tau}_{1} \times \boldsymbol{\tau}_{2}}{\left\|\boldsymbol{\tau}_{1} \times \boldsymbol{\tau}_{2}\right\|},
$$

where $\times$ stands for the cross product of two vectors in $\mathcal{V}$, and \|\| represents the Euclidean norm of a vector in $\mathcal{V}$. We choose $\boldsymbol{n}$ so as to point into the avalanche body. We denote by $-s_{1},-s_{2}$ and $c(c>0)$ the components of $\boldsymbol{n}$ with respect to the Cartesian basis of $\mathcal{V}$ associated to $O x_{1} x_{2} x_{3}$, collect $s_{1}, s_{2}$ in the 2-column matrix

$$
\mathbf{s} \equiv\left(s_{1}, s_{2}\right)^{T},
$$

and note that

$$
c=(1+\operatorname{grad} b \cdot \operatorname{grad} b)^{-1 / 2}, \quad \mathbf{s}=c \operatorname{grad} b,
$$

where grad is the gradient operator with respect to the Cartesian coordinates $x_{1}, x_{2}$. Then, the coefficients of the first fundamental form of $\mathcal{S}$ are $\phi_{\alpha \beta} \equiv \boldsymbol{\tau}_{\alpha} \cdot \boldsymbol{\tau}_{\beta}$, and

$$
\left(\phi_{\alpha \beta}\right)=\mathbf{M}_{0}^{-1}, \quad \mathbf{M}_{0} \equiv \mathbf{F}^{-1}(\mathbf{I}-\mathbf{s} \otimes \mathbf{s}) \mathbf{F}^{-T},
$$

holds, where $\mathbf{I}$ is the $2 \times 2$ unit matrix. By the representation

$$
\frac{\partial \boldsymbol{n}}{\partial \xi^{\beta}}=-b_{\alpha \beta} \boldsymbol{\tau}^{\alpha}=-W_{\beta}^{\alpha} \boldsymbol{\tau}_{\alpha}, \quad \beta \in\{1,2\},
$$

with $\left\{\boldsymbol{\tau}^{1}, \boldsymbol{\tau}^{2}\right\}$ the reciprocal basis of $\left\{\boldsymbol{\tau}_{1}, \boldsymbol{\tau}_{2}\right\}$, one defines the coefficients $b_{\alpha \beta}$ of the second fundamental form of $\mathcal{S}$, and the entries $W_{\beta}^{\alpha}$ of the Weingarten matrix. Note the relation

$$
\mathbf{W}=\mathbf{M}_{0} \mathbf{H}, \quad \mathbf{H} \equiv\left(b_{\alpha \beta}\right), \quad \mathbf{W} \equiv\left(W_{\beta}^{\alpha}\right) .
$$


Finally, the curvature tensor $\mathcal{H}$ and the mean curvature $\Omega$ of the surface at a given point on $\mathcal{S}$ are defined by

$$
\mathcal{H} \equiv b_{\alpha \beta} \boldsymbol{\tau}^{\alpha} \otimes \boldsymbol{\tau}^{\beta}=W_{\beta}^{\alpha} \boldsymbol{\tau}_{\alpha} \otimes \boldsymbol{\tau}^{\beta}, \quad \Omega \equiv \frac{1}{2} \operatorname{tr} \mathcal{H}=\frac{1}{2} \operatorname{tr} \mathbf{W} .
$$

Now, if $\boldsymbol{r}$ is the position vector of a point $P \in \mathcal{E}$ lying above the basal surface, the relation

$$
\boldsymbol{r}\left(x_{1}, x_{2}, x_{3}\right)=\boldsymbol{\rho}\left(\xi^{1}, \xi^{2}\right)+\xi \boldsymbol{n}\left(\xi^{1}, \xi^{2}\right) \equiv \boldsymbol{r}\left(\xi^{1}, \xi^{2}, \xi\right), \quad \xi>0
$$

defines a change of coordinates in a neighborhood of $\mathcal{S}$, on condition that the Jacobian $J$ of the transformation (2.3) is non-zero. Since $J$ is given by

$$
J=\frac{1}{c} \operatorname{det} \mathbf{B}, \quad \mathbf{B} \equiv \mathbf{F}(\mathbf{I}-\xi \mathbf{W})
$$

implying

$$
J=J_{0} \operatorname{det}(\mathbf{I}-\xi \mathbf{W}),\left.\quad J_{0} \equiv J\right|_{\xi=0}=\frac{1}{c} \operatorname{det} \mathbf{F} \neq 0,
$$

the condition $J \neq 0$ is equivalent to

$$
\operatorname{det}(\mathbf{I}-\xi \mathbf{W}) \neq 0,
$$

which we assume to be valid in the domain occupied by the avalanche mass, and which exactly defines what is meant by "arbitrary" topography in this paper and in related works, e.g., Bouchut and Westdickenberg [1], Luca et al. [10], [11]. The vectors

$$
\boldsymbol{g}_{\beta} \equiv \frac{\partial \boldsymbol{r}}{\partial \xi^{\beta}}=\left(\delta^{\alpha}{ }_{\beta}-\xi W_{\beta}^{\alpha}\right) \boldsymbol{\tau}_{\alpha}, \quad \beta \in\{1,2\}, \quad \boldsymbol{g}_{3} \equiv \frac{\partial \boldsymbol{r}}{\partial \xi}=\boldsymbol{n},
$$

where $\delta_{\beta}^{\alpha}$ is the Kronecker symbol, define the natural basis of $\mathcal{V}$ at the point $P$. Clearly, $\boldsymbol{g}_{1}, \boldsymbol{g}_{2}$ are parallel to $\mathcal{S}$, and $\boldsymbol{g}_{3}$ is normal to $\mathcal{S}$. Thus, in the representation of a vector $\boldsymbol{w} \in \mathcal{V}$ with respect to this basis we can identify a tangential component $\boldsymbol{w}_{\tau}$ and a normal component $\boldsymbol{w}_{n}$, that is,

$$
\boldsymbol{w}=\underbrace{w^{1} \boldsymbol{g}_{1}+w^{2} \boldsymbol{g}_{2}}_{\boldsymbol{w}_{\tau}}+\underbrace{w^{3} \boldsymbol{g}_{3}}_{\boldsymbol{w}_{n}}, \quad \boldsymbol{w}_{\tau} \equiv w^{\beta} \boldsymbol{g}_{\beta}=\boldsymbol{w}-\boldsymbol{w}_{n}, \quad \boldsymbol{w}_{n} \equiv w^{3} \boldsymbol{g}_{3}=(\boldsymbol{w} \cdot \boldsymbol{n}) \boldsymbol{n} .
$$

We close this section by introducing the notation

$$
\mathbf{M} \equiv \mathbf{B}^{-1}(\mathbf{I}-\mathbf{s} \otimes \mathbf{s}) \mathbf{B}^{-T},
$$

and mentioning that

$$
\mathbf{M}_{0}=\left.\mathbf{M}\right|_{\xi=0}, \quad \mathbf{M}=(\mathbf{I}-\xi \mathbf{W})^{-1} \mathbf{M}_{0}(\mathbf{I}-\xi \mathbf{W})^{-T} .
$$

The matrix $\mathbf{M}$ enters the approach via the metric tensor corresponding to the transformation (2.3), see Bouchut and Westdickenberg [1]. That is, with $g_{i j} \equiv \boldsymbol{g}_{i} \cdot \boldsymbol{g}_{j}$, we have

$$
\left(g_{i j}\right)=\left(\begin{array}{cc}
\mathbf{M}^{-1} & \mathbf{0} \\
\mathbf{0} & 1
\end{array}\right)
$$




\section{Model equations}

We model the avalanche body by a binary mixture consisting of a solid and a fluid. In doing so, we adopt Truesdell's mixture theory, see Truesdell [30]. In particular, it is therefore assumed that each constituent is a continuum in its own right, and that the configurations of the two continua are superposed at each moment. So, the phase separation is not accounted for. To identify the quantities referring to the two constituents we use the indices $s$ (solid) and $f$ (fluid). Thus, $\rho_{s}$ and $\rho_{f}$ denote the partial densities (mass of a constituent per volume of the mixture), while $\boldsymbol{\sigma}_{s}$ and $\boldsymbol{\sigma}_{f}$ are the constituent stress tensors. However, instead of $\boldsymbol{v}_{s}, \boldsymbol{v}_{f}$ for the constituent velocities, we use $\boldsymbol{v}$ and $\boldsymbol{u}$, respectively. Then, assuming that no mass interactions are present, for the solid constituent the mass and momentum balance equations in an inertial reference frame can be written as

$$
\frac{\partial \rho_{s}}{\partial t}+\operatorname{div}\left(\rho_{s} \boldsymbol{v}\right)=0, \quad \frac{\partial \rho_{s} \boldsymbol{v}}{\partial t}+\operatorname{div}\left(\rho_{s} \boldsymbol{v} \otimes \boldsymbol{v}-\boldsymbol{\sigma}_{s}\right)=\rho_{s} \boldsymbol{b}+\stackrel{+}{\boldsymbol{m}},
$$

where $\boldsymbol{b}$ is the specific body force, $\stackrel{+}{\boldsymbol{m}}$ is the interaction force, and div is the spatial divergence operator. Similarly, for the fluid constituent we have

$$
\frac{\partial \rho_{f}}{\partial t}+\operatorname{div}\left(\rho_{f} \boldsymbol{u}\right)=0, \quad \frac{\partial \rho_{f} \boldsymbol{u}}{\partial t}+\operatorname{div}\left(\rho_{f} \boldsymbol{u} \otimes \boldsymbol{u}-\boldsymbol{\sigma}_{f}\right)=\rho_{f} \boldsymbol{b}-\stackrel{+}{\boldsymbol{m}} .
$$

Taking the opposite of $\stackrel{+}{\boldsymbol{m}}$ as interaction force in (3.2), Truesdell's third metaphysical principle (i.e., the mixture as a whole must be described by the same laws which apply for a single body) is fulfilled.

We assume that the constituents are density preserving, that is the true densities $\tilde{\rho}_{s}, \tilde{\rho}_{f}$ (mass of a constituent per volume of that constituent) are constant in time and uniform in space. Moreover, we suppose that there is no void space within the mixture, that is

$$
\nu_{s}+\nu_{f}=1,
$$

where $\nu_{s}, \nu_{f}$ are the constituent volume fractions (constituent volume per mixture volume). We have

$$
\rho_{s}=\nu_{s} \tilde{\rho}_{s}, \quad \rho_{f}=\nu_{f} \tilde{\rho}_{f},
$$

or, simplifying the notation with the aid of $\nu \equiv \nu_{s}$,

$$
\rho_{s}=\nu \tilde{\rho}_{s}, \quad \rho_{f}=(1-\nu) \tilde{\rho}_{f},
$$

which turns equations (3.1), (3.2) into

$$
\frac{\partial \nu}{\partial t}+\operatorname{div}(\nu \boldsymbol{v})=0, \quad \frac{\partial \nu \boldsymbol{v}}{\partial t}+\operatorname{div}\left\{\nu \boldsymbol{v} \otimes \boldsymbol{v}-\frac{1}{\tilde{\rho}_{s}} \boldsymbol{\sigma}_{s}\right\}=\nu \boldsymbol{b}+\frac{1}{\tilde{\rho}_{s}} \stackrel{+}{\boldsymbol{m}}
$$

and

$$
\begin{gathered}
\frac{\partial(1-\nu)}{\partial t}+\operatorname{div}\{(1-\nu) \boldsymbol{u}\}=0 \\
\frac{\partial(1-\nu) \boldsymbol{u}}{\partial t}+\operatorname{div}\left\{(1-\nu) \boldsymbol{u} \otimes \boldsymbol{u}-\frac{1}{\tilde{\rho}_{f}} \boldsymbol{\sigma}_{f}\right\}=(1-\nu) \boldsymbol{b}-\frac{1}{\tilde{\rho}_{f}} \stackrel{+}{\boldsymbol{m}},
\end{gathered}
$$


respectively. Note that $\nu \in(0,1)$, as the constituents are assumed to simultaneously occupy the same region in $\mathcal{E}$.

Equations (3.4), (3.5) hold in the domain occupied by the avalanche mass, which is bounded below by the bed surface and above by the free surface. However, the free surface is unknown, and hence, e.g., an evolution equation for it is needed. Here we consider that the free surface

$$
F\left(x_{1}, x_{2}, x_{3}, t\right)=0
$$

is material for each constituent, which yields the so-called kinematic boundary conditions

$$
\frac{\partial F}{\partial t}+\nabla F \cdot \boldsymbol{v}=0, \quad \frac{\partial F}{\partial t}+\nabla F \cdot \boldsymbol{u}=0 \quad \text { at } \quad F=0,
$$

where $\nabla$ is the spatial gradient operator. Relations (3.7) are equivalent to

$$
\frac{\partial F}{\partial t}+\nabla F \cdot \boldsymbol{v}=0, \quad \nabla F \cdot(\boldsymbol{v}-\boldsymbol{u})=0 \quad \text { at } \quad F=0,
$$

which shows, in particular, that the projections of the velocities $\boldsymbol{v}, \boldsymbol{u}$ (evaluated on the free surface) onto the normal direction to the free surface are required to be equal. This condition is approximately satisfied if $\boldsymbol{v}-\boldsymbol{u} \approx \boldsymbol{0}$, but for "large" $\boldsymbol{v}-\boldsymbol{u}$ it is less realistic, as Pitman and Le [18] (who also used (3.7)) noticed. However, the account of (3.7) brings considerable simplifications in the averaging procedure, see the forthcoming Proposition 5.1. On the other hand, since each constituent of the mixture is assumed to be a continuum body in its own right, the free surface corresponding to each constituent has to be a material surface, see Truesdell and Toupin [29], p. 509; with a common free surface for both constituents we then obviously have (3.7).

Now, equations (3.4), (3.5) are complemented by boundary conditions at the bottom topography and at the free surface, as follows. The velocity of each constituent is supposed to be tangent to the basal topography,

$$
\boldsymbol{v} \cdot \boldsymbol{n}=0, \quad \boldsymbol{u} \cdot \boldsymbol{n}=0,
$$

which expresses the so-called non-penetration condition, and both solid and fluid stresses normal to the free surface are taken to be zero, that is,

$$
\boldsymbol{\sigma}_{s} \boldsymbol{n}_{F} \cdot \boldsymbol{n}_{F}=0, \quad \boldsymbol{\sigma}_{f} \boldsymbol{n}_{F} \cdot \boldsymbol{n}_{F}=0 \quad \text { at } \quad F=0,
$$

where $\boldsymbol{n}_{F}$ denotes a unit normal vector field to the surface $F=0$. It is worthwhile to mention that, in avalanche modeling the free surface is usually assumed traction-free, which, for the case presented here, means

$$
\boldsymbol{\sigma}_{s} \boldsymbol{n}_{F}=\boldsymbol{O}, \quad \boldsymbol{\sigma}_{f} \boldsymbol{n}_{F}=\boldsymbol{O} \quad \text { at } \quad F=0 .
$$

However, as shown in Luca, Tai, Kuo [10] for the one-component avalanche mass, conditions (3.10) could lead to an overdetermined system of equations. Later on, in section 6 , we merely assume that the stresses on the free surface are negligibly small, see (6.5) below.

The unknown fields in (3.4)-(3.9) are $\nu, \boldsymbol{v}, \boldsymbol{u}, F, \boldsymbol{\sigma}_{s}, \boldsymbol{\sigma}_{f}, \stackrel{+}{\boldsymbol{m}}$, and since there are 10 scalar equations, viz. (3.4), (3.5), (3.7), we need closure relations in terms of 10 
basic scalar fields. We take 8 of these basic unknowns to be $\nu, \boldsymbol{v}, \boldsymbol{u}, F$. Then, we represent the stress tensors $\boldsymbol{\sigma}_{s}, \boldsymbol{\sigma}_{f}$ as

$$
\boldsymbol{\sigma}_{s}=-p_{s} \boldsymbol{1}+\boldsymbol{\sigma}_{s}^{E}, \quad \boldsymbol{\sigma}_{f}=-p_{f} \boldsymbol{1}+\boldsymbol{\sigma}_{f}^{E}
$$

where the identification of the constituent pressures $p_{s}, p_{f}$ and of the extra stress tensors $\boldsymbol{\sigma}_{s}^{E}, \boldsymbol{\sigma}_{f}^{E}$ has to be made clear when giving the closure relations for $\boldsymbol{\sigma}_{s, f}$, and take $p_{s}, p_{f}$ as the other 2 independent variables. (We note that the trace of $\boldsymbol{\sigma}_{s, f}^{E}$ in (3.11) is not necessarily zero.) Thus, the extra stresses $\boldsymbol{\sigma}_{s, f}^{E}$ and the interaction force $\stackrel{+}{\boldsymbol{m}}$ have to be given in terms of these basic fields.

However, to look for a solution of the system of equations and boundary conditions (3.4)-(3.9), complemented by closure relations for $\boldsymbol{\sigma}_{s, f}^{E}, \stackrel{+}{\boldsymbol{m}}$ and initial data, is rather a challenging task. To simplify the formulated problem we use the depth-averaging procedure as described in section 5. Essentially, the approach is the same as that used in Luca et al. [11] for the one-component avalanche mass, and is based on equations (3.4)-(3.9) written in the curvilinear coordinates of section 2 .

For the forthcoming sections $4-7$ the specification of $p_{s, f}$ and $\boldsymbol{\sigma}_{s, f}^{E}$ in (3.11) is not important, but we can keep in mind that, in the models proposed in section 8 , we have $p_{s} \equiv-\boldsymbol{\sigma}_{s} \boldsymbol{n} \cdot \boldsymbol{n}$, and $\boldsymbol{\sigma}_{f}$ is given by a Newtonian/non-Newtonian law, see (8.1) below. We may think of $p_{s}, p_{f}$ as independent variables required by the saturation constraint (3.3), and by the constraint that both constituents have the same free surface, see $(3.7)$.

\section{Non-dimensional model equations in curvilinear coordinates}

Now we want to write equations (3.4)-(3.9) in terms of the contravariant components $v^{i}, u^{i}, T_{s}^{i j}, T_{f}^{i j}, b^{i}, \stackrel{+}{m}^{i}(i, j=1,2,3)$ of $\boldsymbol{v}, \boldsymbol{u}, \boldsymbol{\sigma}_{s}^{E}, \boldsymbol{\sigma}_{f}^{E}, \boldsymbol{b}$ and $\stackrel{+}{\boldsymbol{m}}$, respectively, relative to the basis $\left\{\boldsymbol{g}_{1}, \boldsymbol{g}_{2}, \boldsymbol{g}_{3}\right\}$ :

$$
\boldsymbol{v}=v^{i} \boldsymbol{g}_{i}, \quad \boldsymbol{u}=u^{i} \boldsymbol{g}_{i}, \quad \boldsymbol{\sigma}_{s}^{E}=T_{s}^{i j} \boldsymbol{g}_{i} \otimes \boldsymbol{g}_{j}, \quad \boldsymbol{\sigma}_{f}^{E}=T_{f}^{i j} \boldsymbol{g}_{i} \otimes \boldsymbol{g}_{j}, \quad \boldsymbol{b}=b^{i} \boldsymbol{g}_{i}, \quad \stackrel{+}{\boldsymbol{m}}=\stackrel{+}{m^{i}} \boldsymbol{g}_{i} .
$$

To this end we first collect these components in block matrix form, as follows:

$$
\begin{gathered}
\mathbf{v} \equiv\left(\begin{array}{c}
v^{1} \\
v^{2}
\end{array}\right), \quad \mathrm{v} \equiv v^{3}, \quad \mathbf{u} \equiv\left(\begin{array}{c}
u^{1} \\
u^{2}
\end{array}\right), \quad \mathrm{u} \equiv u^{3}, \quad\left(\begin{array}{cc}
\mathbf{P}_{s} & \mathbf{p}_{s} \\
\mathbf{p}_{s}^{T} & T_{s}^{33}
\end{array}\right) \equiv\left(T_{s}^{i j}\right), \\
\left(\begin{array}{cc}
\mathbf{P}_{f} & \mathbf{p}_{f} \\
\mathbf{p}_{f}^{T} & T_{f}^{33}
\end{array}\right) \equiv\left(T_{f}^{i j}\right), \quad \mathbf{b} \equiv\left(\begin{array}{c}
b^{1} \\
b^{2}
\end{array}\right), \quad \mathrm{b} \equiv b^{3}, \quad \stackrel{+}{\mathbf{m}} \equiv\left(\begin{array}{c}
\stackrel{+}{m}^{1} \\
\dot{+}^{2}
\end{array}\right), \quad \stackrel{+}{\mathrm{m}} \equiv \stackrel{+}{m}^{3} .
\end{gathered}
$$

In the above, $\mathbf{P}_{s}$ and $\mathbf{P}_{f}$ are $2 \times 2$ matrices, and $\mathbf{p}_{s}, \mathbf{p}_{f}$ are 2 -column matrices. Then, we introduce the (two-dimensional) Grad and Div operators by

$$
\operatorname{Grad} f \equiv\left(\frac{\partial f}{\partial \xi^{1}}, \frac{\partial f}{\partial \xi^{2}}\right)^{T}, \operatorname{Grad} \mathbf{w} \equiv\left(\frac{\partial w^{\alpha}}{\partial \xi^{\beta}}\right), \operatorname{Div} \mathbf{w} \equiv \frac{\partial w^{\alpha}}{\partial \xi^{\alpha}}, \operatorname{Div} \mathbf{P} \equiv\left(\frac{\partial P^{1 \beta}}{\partial \xi^{\beta}}, \frac{\partial P^{2 \beta}}{\partial \xi^{\beta}}\right)^{T}
$$

where $f, \mathbf{w} \equiv\left(w^{1}, w^{2}\right)^{T}$ and $\mathbf{P} \equiv\left(P^{\alpha \beta}\right)_{\alpha, \beta \in\{1,2\}}$ are smooth fields on $\Delta_{0}$. Finally, assuming that $\xi^{1}, \xi^{2}$ are length dimensional coordinates, and that the free surface equation (3.6) emerges as $\xi=h(\boldsymbol{\xi}, t)$ under transformation (2.3), we switch to non- 
dimensional variables according to the scalings

$$
\begin{gathered}
\left(x_{1}, x_{2}, x_{3}, t\right)=L\left(\hat{x}_{1}, \hat{x}_{2}, \hat{x}_{3}, \hat{t} / \sqrt{L g}\right), \quad\left(\xi^{1}, \xi^{2}, \xi\right)=L\left(\hat{\xi}^{1}, \hat{\xi}^{2}, \hat{\xi}\right), \quad(b, h)=L(\hat{b}, \hat{h}), \\
(\mathbf{v}, \mathrm{v}, \mathbf{u}, \mathbf{u})=\sqrt{L g}(\hat{\mathbf{v}}, \hat{\mathrm{v}}, \hat{\mathbf{u}}, \hat{\mathbf{u}}), \quad(\mathbf{b}, \mathrm{b})=g(\hat{\mathbf{b}}, \hat{\mathrm{b}}), \quad(\stackrel{+}{\mathbf{m}}, \stackrel{+}{\mathrm{m}})=\tilde{\rho}_{s} g(\stackrel{\hat{\mathbf{m}}}{\stackrel{\hat{+}}{\mathrm{m}})}, \\
\left(p_{s}, T_{s}^{i j}\right)=\tilde{\rho}_{s} L g\left(\hat{p}_{s}, \hat{T}_{s}^{i j}\right), \quad\left(p_{f}, T_{f}^{i j}\right)=\tilde{\rho}_{f} L g\left(\hat{p}_{f}, \hat{T}_{f}^{i j}\right),
\end{gathered}
$$

where $L$ is a typical length tangent to the topography, and $g$ is the constant gravitational acceleration. Then, the non-dimensional form of equations and boundary conditions (3.4)-(3.9) in the curvilinear coordinates defined in section 2 can be simply carried over from Luca et al. [10], see Proposition 3 therein, with only minor modifications ${ }^{1}$. Thus, omitting the hat and using the notations

$$
\begin{gathered}
\boldsymbol{\Gamma}(-p \mathbf{M}, \mathbf{0}) \equiv p\left\{\mathbf{B}^{-1} \frac{\partial \mathbf{B}}{\partial \xi^{\alpha}} \mathbf{M} \mathbf{e}_{\alpha}+\operatorname{tr}\left(\mathbf{W}(\mathbf{I}-\xi \mathbf{W})^{-1}\right) \mathbf{B}^{-1} \mathbf{s}\right\} \\
\boldsymbol{\Gamma}(\mathbf{P}, \mathbf{p}) \equiv-\mathbf{B}^{-1} \frac{\partial \mathbf{B}}{\partial \xi^{\alpha}} \mathbf{P} \mathbf{e}_{\alpha}+2 \mathbf{B}^{-1} \mathbf{F} \mathbf{W} \mathbf{p}+\Gamma(\mathbf{P}) \mathbf{B}^{-1} \mathbf{s} \\
\boldsymbol{\Gamma}(\mathbf{w}, \mathbf{w}) \equiv-\mathbf{B}^{-1} \frac{\partial \mathbf{B}}{\partial \xi^{\alpha}}(\mathbf{w} \otimes \mathbf{w}) \mathbf{e}_{\alpha}+2 \mathrm{w}^{-1} \mathbf{F W} \mathbf{w}+\Gamma(\mathbf{w}) \mathbf{B}^{-1} \mathbf{s}, \\
\Gamma(\mathbf{P}) \equiv-\mathbf{B}^{T} \mathbf{F}^{-T} \mathbf{H} \cdot \mathbf{P}, \quad \Gamma(\mathbf{w}) \equiv-\mathbf{B}^{T} \mathbf{F}^{-T} \mathbf{H} \cdot(\mathbf{w} \otimes \mathbf{w}),
\end{gathered}
$$

where $p$, w are scalars, $\mathbf{p}, \mathbf{w}$ are 2 -column matrices, $\mathbf{P}$ is a $2 \times 2$ matrix, and $\mathbf{e}_{1} \equiv(1,0)^{T}$, $\mathbf{e}_{2} \equiv(0,1)^{T}$, as well as

$$
c_{0} \equiv \tilde{\rho}_{s} / \tilde{\rho}_{f},
$$

we deduce the follwoing statements.

Proposition 4.1. In the curvilinear coordinates (2.3) and in non-dimensional form, the balance equations (3.4) corresponding to the solid constituent emerge as

$$
\begin{gathered}
\frac{\partial J \nu}{\partial t}+\operatorname{Div}\{J \nu \mathbf{v}\}+\frac{\partial}{\partial \xi}\{J \nu \mathbf{v}\}=0 \\
\frac{\partial}{\partial t}\{J \nu \mathbf{v}\}+\operatorname{Div}\left\{J\left(\nu \mathbf{v} \otimes \mathbf{v}+p_{s} \mathbf{M}-\mathbf{P}_{s}\right)\right\}+\frac{\partial}{\partial \xi}\left\{J\left(\nu \mathbf{v} \mathbf{v}-\mathbf{p}_{s}\right)\right\} \\
+J \mathbf{\Gamma}\left(-p_{s} \mathbf{M}, \mathbf{0}\right)+J \boldsymbol{\Gamma}\left(\mathbf{P}_{s}, \mathbf{p}_{s}\right) \\
=J \nu \mathbf{b}+J \nu \boldsymbol{\Gamma}(\mathbf{v}, \mathrm{v})+J \stackrel{+}{\mathbf{m}}, \\
\frac{\partial}{\partial t}\{J \nu \mathbf{v}\}+\operatorname{Div}\left\{J\left(\nu \mathbf{v} \mathbf{v}-\mathbf{p}_{s}\right)\right\}+\frac{\partial}{\partial \xi}\left\{J\left(\nu \mathrm{v}^{2}-T_{s}^{33}\right)\right\}+J \frac{\partial p_{s}}{\partial \xi}+J \Gamma\left(\mathbf{P}_{s}\right) \\
=J \nu \mathrm{b}+J \nu \Gamma(\mathbf{v})+J \stackrel{+}{\mathrm{m}},
\end{gathered}
$$

\footnotetext{
${ }^{1}$ To avoid confusion, we draw attention to the fact that $\mathbf{P}, \mathbf{p}$ and $T^{33}$ are used in Luca et al. [10], [11] to denote the contravariant components of the stress tensor.
} 
while the balance equations (3.5) corresponding to the fluid constituent turn into

$$
\begin{gathered}
\frac{\partial}{\partial t}\{J(1-\nu)\}+\operatorname{Div}\{J(1-\nu) \mathbf{u}\}+\frac{\partial}{\partial \xi}\{J(1-\nu) \mathrm{u}\}=0 \\
\frac{\partial}{\partial t}\{J(1-\nu) \mathbf{u}\}+\operatorname{Div}\left\{J\left[(1-\nu) \mathbf{u} \otimes \mathbf{u}+p_{f} \mathbf{M}-\mathbf{P}_{f}\right]\right\}+\frac{\partial}{\partial \xi}\left\{J\left[(1-\nu) \mathbf{u}-\mathbf{p}_{f}\right]\right\} \\
+J \boldsymbol{\Gamma}\left(-p_{f} \mathbf{M}, \mathbf{0}\right)+J \boldsymbol{\Gamma}\left(\mathbf{P}_{f}, \mathbf{p}_{f}\right)=J(1-\nu) \mathbf{b}+J(1-\nu) \boldsymbol{\Gamma}(\mathbf{u}, \mathrm{u})-c_{0} J \stackrel{+}{\mathbf{m}} \\
\left.\frac{\partial}{\partial t}\{J(1-\nu) \mathrm{u}\}+\operatorname{Div}\left\{J\left[(1-\nu) \mathrm{u} \mathbf{u}-\mathbf{p}_{f}\right]\right\}+\frac{\partial}{\partial \xi}\left\{J\left[(1-\nu) \mathrm{u}^{2}-T_{f}^{33}\right)\right]\right\} \\
+J \frac{\partial p_{f}}{\partial \xi}+J \Gamma\left(\mathbf{P}_{f}\right)=J(1-\nu) \mathrm{b}+J(1-\nu) \Gamma(\mathbf{u})-c_{0} J \stackrel{+}{\mathrm{m}}
\end{gathered}
$$

Moreover, the kinematic boundary conditions (3.7) appear as

$$
\frac{\partial h}{\partial t}+\operatorname{Grad} h \cdot \mathbf{v}=\mathrm{v}, \quad \frac{\partial h}{\partial t}+\operatorname{Grad} h \cdot \mathbf{u}=\mathrm{u} \quad \text { at } \quad \xi=h(\boldsymbol{\xi}, t),
$$

the conditions (3.8) of the tangency of the velocities emerge as

$$
\mathrm{v}=0, \quad \mathrm{u}=0 \quad \text { at } \quad \xi=0
$$

and the dynamic conditions (3.9) become

$$
\begin{aligned}
& \left\{\left(-p_{s} \mathbf{M}+\mathbf{P}_{s}\right) \operatorname{Grad} h-\mathbf{p}_{s}\right\} \cdot \operatorname{Grad} h-\left(\mathbf{p}_{s} \cdot \operatorname{Grad} h+p_{s}-T_{s}^{33}\right)=0, \\
& \left\{\left(-p_{f} \mathbf{M}+\mathbf{P}_{f}\right) \operatorname{Grad} h-\mathbf{p}_{f}\right\} \cdot \operatorname{Grad} h-\left(\mathbf{p}_{f} \cdot \operatorname{Grad} h+p_{f}-T_{f}^{33}\right)=0
\end{aligned}
$$

at $\xi=h(\boldsymbol{\xi}, t)$.

Equations (4.8), (4.11) are referred to as tangential momentum balance equations, and (4.9), (4.12) are called normal momentum balance equations. The $\Gamma, \Gamma$ terms appear as source terms in (4.8), (4.9), (4.11), (4.12), due to the Christoffel symbols corresponding to the change of coordinates. In the next section we outline the way by which we exploit the equations and boundary conditions given in Proposition 4.1.

\section{Depth-averaging procedure}

Now we explain the depth-integration procedure, which we use to derive the final modeling equations. First, the mass balance equations (4.7), (4.10) and the tangential momentum balance equations (4.8), (4.11) are integrated with respect to the normal variable $\xi$ from 0 to $h(\boldsymbol{\xi}, t)$. So, by using the Leibniz rules

$$
\begin{gathered}
\int_{0}^{h(\boldsymbol{\xi}, t)} \operatorname{Div} \mathbf{w} d \xi=\operatorname{Div} \int_{0}^{h(\boldsymbol{\xi}, t)} \mathbf{w} d \xi-\mathbf{w}(\boldsymbol{\xi}, h(\boldsymbol{\xi}, t)) \cdot \operatorname{Grad} h, \\
\int_{0}^{h(\boldsymbol{\xi}, t)} \operatorname{Div} \mathbf{P} d \xi=\operatorname{Div} \int_{0}^{h(\boldsymbol{\xi}, t)} \mathbf{P} d \xi-\mathbf{P}(\boldsymbol{\xi}, h(\boldsymbol{\xi}, t)) \operatorname{Grad} h,
\end{gathered}
$$

that hold for a 2-column matrix $\mathbf{w}(\boldsymbol{\xi}, \xi, t)$ and for a square matrix $\mathbf{P}(\boldsymbol{\xi}, \xi, t)$ of order 2 , as well as the boundary conditions (4.14), (4.13), we deduce the following. 
Proposition 5.1. For the solid constituent, the depth-integrated mass balance and tangential momentum balance equations are given by

$$
\begin{gathered}
\frac{\partial}{\partial t} \int_{0}^{h(\boldsymbol{\xi}, t)} J \nu d \xi+\operatorname{Div} \int_{0}^{h(\boldsymbol{\xi}, t)} J \nu \mathbf{v} d \xi=0 \\
\frac{\partial}{\partial t} \int_{0}^{h(\boldsymbol{\xi}, t)} J \nu \mathbf{v} d \xi+\operatorname{Div} \int_{0}^{h(\boldsymbol{\xi}, t)} J\left\{\nu \mathbf{v} \otimes \mathbf{v}+p_{s} \mathbf{M}-\mathbf{P}_{s}\right\} d \xi+\left.\left(J \mathbf{p}_{s}\right)\right|_{\xi=0} \\
\quad+\left\{J\left[\left(-p_{s} \mathbf{M}+\mathbf{P}_{s}\right) \operatorname{Grad} h-\mathbf{p}_{s}\right]\right\}_{\xi=h}+\int_{0}^{h(\boldsymbol{\xi}, t)} J\left\{\boldsymbol{\Gamma}\left(-p_{s} \mathbf{M}, \mathbf{0}\right)+\boldsymbol{\Gamma}\left(\mathbf{P}_{s}, \mathbf{p}_{s}\right)\right\} d \xi \\
=\int_{0}^{h(\boldsymbol{\xi}, t)} J \nu \mathbf{b} d \xi+\int_{0}^{h(\boldsymbol{\xi}, t)} J \nu \mathbf{\Gamma}(\mathbf{v}, \mathbf{v}) d \xi+\int_{0}^{h(\boldsymbol{\xi}, t)} J \stackrel{+}{\mathbf{m}} d \xi
\end{gathered}
$$

Similarly, for the fluid constituent we have

$$
\begin{gathered}
\frac{\partial}{\partial t} \int_{0}^{h(\boldsymbol{\xi}, t)} J(1-\nu) d \xi+\operatorname{Div} \int_{0}^{h(\boldsymbol{\xi}, t)} J(1-\nu) \mathbf{u} d \xi=0, \\
\frac{\partial}{\partial t} \int_{0}^{h(\boldsymbol{\xi}, t)} J(1-\nu) \mathbf{u} d \xi+\operatorname{Div} \int_{0}^{h(\boldsymbol{\xi}, t)} J\left\{(1-\nu) \mathbf{u} \otimes \mathbf{u}+p_{f} \mathbf{M}-\mathbf{P}_{f}\right\} d \xi \\
+\left.\left(J \mathbf{p}_{f}\right)\right|_{\xi=0}+\left\{J\left[\left(-p_{f} \mathbf{M}+\mathbf{P}_{f}\right) \operatorname{Grad} h-\mathbf{p}_{f}\right]\right\}_{\xi=h} \\
+\int_{0}^{h(\boldsymbol{\xi}, t)} J\left\{\boldsymbol{\Gamma}\left(-p_{f} \mathbf{M}, \mathbf{0}\right)+\boldsymbol{\Gamma}\left(\mathbf{P}_{f}, \mathbf{p}_{f}\right)\right\} d \xi \\
=\int_{0}^{h(\boldsymbol{\xi}, t)} J(1-\nu) \mathbf{b} d \xi+\int_{0}^{h(\boldsymbol{\xi}, t)} J(1-\nu) \boldsymbol{\Gamma}(\mathbf{u}, \mathrm{u}) d \xi-c_{0} \int_{0}^{h(\boldsymbol{\xi}, t)} J \stackrel{+}{\mathbf{m}} d \xi .
\end{gathered}
$$

Then we note that, assuming that the mass balance equation (4.7) and boundary condition $(4.14)_{1}$ hold, the kinematic boundary condition $(4.13)_{1}$ is equivalent to the depth-integrated mass balance equation (5.1). A similar statement is valid for the fluid constituent. Consequently, the system of equations and boundary conditions (4.7)-(4.15) is equivalent to (4.7)-(4.12), (5.1), (5.3), (4.14), (4.15). Next, instead of one of the equivalent systems mentioned above, we use the system consisting of

$$
\text { (4.7), (5.2), (4.9), (4.10), (5.4), (4.12), (5.1), (5.3), (4.14), (4.15), }
$$

which is a consequence of (4.7)-(4.15). The idea is to use (5.5) under some ordering approximations to deduce a set of equations involving only the depth-average of the field quantities entering equations (5.5), as we subsequently describe. Thus, by using the mass balance equations (4.7), (4.10) and the boundary conditions (4.14), one deduces the normal velocities $\mathrm{v}, \mathrm{u}$ as

$$
\begin{gathered}
\mathrm{v}=-\frac{1}{J \nu} \int_{0}^{\xi}\left\{\frac{\partial J \nu}{\partial t}+\operatorname{Div}(J \nu \mathbf{v})\right\} d \xi^{\prime}, \\
\mathrm{u}=-\frac{1}{J(1-\nu)} \int_{0}^{\xi}\left\{\frac{\partial J(1-\nu)}{\partial t}+\operatorname{Div}(J(1-\nu) \mathbf{u})\right\} d \xi^{\prime}
\end{gathered}
$$


which will be substituted into (5.2), (4.9), (5.4), (4.12). Then, by using the closure relation for the interaction force $\stackrel{+}{\boldsymbol{m}}$ and scaling approximations, from $(4.9),(4.12)$ and (4.15) we obtain the constituent pressures $p_{s}, p_{f}$, the expressions of which are next introduced into (5.2), (5.4). Finally, the depth-integrated equations (5.1)-(5.4) are transformed into equations for the depth-averaged solid volume fraction, the depthaveraged tangential velocities, and for the avalanche depth, by closing them with constitutive relations and flow rules that account for the shallowness of the flowing material layer.

The benefit of the averaging procedure, which has already been used, e.g, in Savage and Hutter [24], Luca et al. [10], [11], Tai and Kuo [28] for the one-component avalanche mass, and in Pitman and Le [18], Pelanti, Bouchut and Mangenay [17] for a solid-fluid mixture, is that the normal variable $\xi$ is eliminated.

\section{Ordering approximations}

We exploit the equations and boundary conditions (5.5) under some scaling approximations, by which physically non-significant contributions of the field variables are neglected. In order to express these approximations we use an aspect ratio $\epsilon \ll 1$ between a typical thickness normal to the topography and a typical length-scale $L$ tangent to the topography, and a constant $\gamma \in(0,1)$. We suppose that the same $L$ is considered to define the non-dimensional quantities in (4.1). For concreteness, we mention that for snow and debris avalanches we usually have $\epsilon \approx 10^{-2}$ and $\gamma \approx \frac{1}{2}$, see Pudasaini and Hutter [22], p.188. We also make use of the definition

$$
\bar{f}(\boldsymbol{\xi}, t) \equiv \frac{1}{h(\boldsymbol{\xi}, t)} \int_{0}^{h(\boldsymbol{\xi}, t)} f(\boldsymbol{\xi}, \xi, t) d \xi
$$

for the mean value (along the depth) or depth-average of a quantity $f$. Thus, we introduce the following approximations, which we shall later comment upon.

a) Geometric approximations: the material layer is thin, i.e., $h=O(\epsilon)$.

b) Flow rule approximations: the velocities $\boldsymbol{v}, \boldsymbol{u}$ are such that $\mathbf{v}=O(1), \mathbf{u}=O(1)$, and assumptions of Boussinesq type hold, viz.,

$$
\begin{gathered}
\int_{0}^{h(\boldsymbol{\xi}, t)} \xi \mathbf{v} d \xi=\frac{1}{2} h^{2} m_{1}^{s} \overline{\mathbf{v}}+O\left(\epsilon^{2+\gamma}\right), \quad \int_{0}^{h(\boldsymbol{\xi}, t)} \mathbf{v} \otimes \mathbf{v} d \xi=h m_{2}^{s} \overline{\mathbf{v}} \otimes \overline{\mathbf{v}}+O\left(\epsilon^{2+\gamma}\right), \\
\int_{0}^{h(\boldsymbol{\xi}, t)} \xi \mathbf{v} \otimes \mathbf{v} d \xi=\frac{1}{2} h^{2} m_{3}^{s} \overline{\mathbf{v}} \otimes \overline{\mathbf{v}}+O\left(\epsilon^{2+\gamma}\right), \quad \int_{0}^{h(\boldsymbol{\xi}, t)} \mathbf{v} \mathbf{v} d \xi=\frac{1}{2} h^{2} \beta_{s} \overline{\mathbf{v}}+O\left(\epsilon^{2+\gamma}\right),
\end{gathered}
$$

and similar relations with scalar coefficients $m_{1}^{f}, m_{2}^{f}, m_{3}^{f}, \beta_{f}$ for the fluid constituent. The momentum correction factors or Boussinesq coefficients $m_{1}^{s}$ to $\beta_{f}$ are supposed to be scalar functions of $\boldsymbol{\xi}, t$ (possibly by means of $\bar{\nu}, \overline{\mathbf{v}}$ ) of order $O(1)$, see Luca et al. [11].

c) Configurational-flow rule approximations: the solid volume fraction $\nu$ and the tangential velocities $\mathbf{v}, \mathbf{u}$ have the properties

$$
\begin{aligned}
& \nu=\bar{\nu}+O(\epsilon), \\
& \int_{0}^{h(\boldsymbol{\xi}, t)} \nu \mathbf{v} d \xi=h n_{1}^{s} \bar{\nu} \overline{\mathbf{v}}+O\left(\epsilon^{2+\gamma}\right), \quad \int_{0}^{h(\boldsymbol{\xi}, t)} \nu \mathbf{v} \otimes \mathbf{v} d \xi=h n_{2}^{s} \bar{\nu} \overline{\mathbf{v}} \otimes \overline{\mathbf{v}}+O\left(\epsilon^{2+\gamma}\right), \\
& \int_{0}^{h(\boldsymbol{\xi}, t)} \nu \mathbf{u} d \xi=h n_{1}^{f} \bar{\nu} \overline{\mathbf{u}}+O\left(\epsilon^{2+\gamma}\right), \quad \int_{0}^{h(\boldsymbol{\xi}, t)} \nu \mathbf{u} \otimes \mathbf{u} d \xi=h n_{2}^{f} \bar{\nu} \overline{\mathbf{u}} \otimes \overline{\mathbf{u}}+O\left(\epsilon^{2+\gamma}\right),
\end{aligned}
$$


where the scalar coefficients $n_{1}^{s}$ to $n_{2}^{f}$ are functions of $\boldsymbol{\xi}, t$ of order $O(1)$.

d) Dynamic stress approximations: corresponding to the motion of the avalanche mass, the stress tensors $\boldsymbol{\sigma}_{s}$ and $\boldsymbol{\sigma}_{f}$ satisfy the conditions

$$
\begin{array}{llll}
p_{s}=O(\epsilon), & \mathbf{P}_{s}=O(\epsilon), & \mathbf{p}_{s}=O\left(\epsilon^{\gamma}\right), & T_{s}^{33}=O\left(\epsilon^{1+\gamma}\right), \\
p_{f}=O(\epsilon), & \mathbf{P}_{f}=O(\epsilon), & \mathbf{p}_{f}=O\left(\epsilon^{\gamma}\right), & T_{f}^{33}=O\left(\epsilon^{1+\gamma}\right) .
\end{array}
$$

Moreover, on the free surface $\xi=h(\boldsymbol{\xi}, t)$ we assume

$$
\left(-p_{s} \mathbf{M}+\mathbf{P}_{s}\right) \operatorname{Grad} h-\mathbf{p}_{s}=O\left(\epsilon^{2+\gamma}\right), \quad\left(-p_{f} \mathbf{M}+\mathbf{P}_{f}\right) \operatorname{Grad} h-\mathbf{p}_{f}=O\left(\epsilon^{2+\gamma}\right) .
$$

We now address assumptions a-d. Thus, by assumption a we consider that the flowing avalanche mass is shallow. In particular, it implies that $\xi=O(\epsilon)$ for $\xi \in[0, h]$, which will be used next.

By assumption b, the tangential velocities of both constituents are significant, while the normal velocities $\mathrm{v}, \mathrm{u}$ are of order $O(\epsilon)$, which can be seen from (5.6) by accounting for $\nu=O(1), \mathbf{v}=O(1), \mathbf{u}=O(1), J=O(1), \xi=O(\epsilon)$. Moreover, postulating (6.1) and similar relations for the fluid constituent, as well as (6.2), tangent velocity profiles $\mathbf{v}, \mathbf{u}$ that slightly deviate from "plug-flow" profiles are envisaged. We explain this by referring to the solid constituent. Thus, inserting $\mathbf{v}=\mathbf{v}(\boldsymbol{\xi}, t)$, which defines a "plug-flow", into the left-hand side of the first three relations in (6.1), we deduce that these relations are satisfied with $m_{1}^{s}=m_{2}^{s}=m_{3}^{s}=1$ and no negligible terms. In view of (5.6), the last assumption in (6.1) actually refers to $\nu$ and $\mathbf{v}$. To see that it is also satisfied by a "plug-flow" of the solid constituent, the volume fraction of which satisfies (6.2), we have first to use

$$
J=J_{0} \operatorname{det}(\mathbf{I}-\xi \mathbf{W})=J_{0}(1-2 \Omega \xi)+O\left(\epsilon^{2}\right)
$$

and $(6.2)$ in $(5.6)_{1}$ to obtain

$$
\mathrm{v}=-\frac{1}{J_{0} \bar{\nu}}\left\{\frac{\partial}{\partial t}\left(J_{0} \bar{\nu}\right)+\operatorname{Div}\left(J_{0} \bar{\nu} \mathbf{v}\right)\right\} \xi+O\left(\epsilon^{2}\right)
$$

and hence

$$
\int_{0}^{h(\boldsymbol{\xi}, t)} \mathbf{v} \mathbf{v} d \xi=-\frac{h^{2}}{2 J_{0} \bar{\nu}}\left\{\frac{\partial}{\partial t}\left(J_{0} \bar{\nu}\right)+\operatorname{Div}\left(J_{0} \bar{\nu} \mathbf{v}\right)\right\} \mathbf{v}+O\left(\epsilon^{3}\right) .
$$

Consequently, noting that $\mathbf{v}=\overline{\mathbf{v}}$, the last relation in (6.1) is satisfied by a "plug-flow" in which (6.2) holds, with the Boussinesq coefficient $\beta_{s}$ given by

$$
\beta_{s}=-\frac{1}{J_{0} \bar{\nu}}\left\{\frac{\partial}{\partial t}\left(J_{0} \bar{\nu}\right)+\operatorname{Div}\left(J_{0} \bar{\nu} \overline{\mathbf{v}}\right)\right\},
$$

and negligible terms of order $O\left(\epsilon^{3}\right)$. It is then clear that the momentum correction factors $m_{1}^{s}$ to $m_{3}^{s}$ distinct from 1 , and $\beta_{s}$ distinct from (6.7) refer to a motion of the avalanche mass different from a "plug-flow" in which (6.2) holds. Corresponding to a power law for the tangential velocity profile, the coefficients $m_{1}^{s}, m_{2}^{s}, m_{3}^{s}$ have been determined in Luca et al. [11], and are shown to belong to $[1,2)$; the coefficient $\beta_{s}$ can be determined as in deducing (6.7).

Assumption $\mathrm{c}$ is suggested by the experimental data recorded in Egashira, Itoh and Takeuchi [5], which show that the solid volume fraction decreases almost linearly 
along the avalanche depth, except near the free surface, where it abruptly goes to zero. As a simplified first approach, we suppose that the solid volume fraction at $(\boldsymbol{\xi}, \xi, t)$ is only slightly different from its depth-averaged value at $(\boldsymbol{\xi}, t)$, see (6.2). Moreover, postulating (6.3), a linear (in $\xi$ ) profile of $\nu$ is envisaged. That is, if $\nu$ is given by the linear profile

$$
\nu=\bar{\nu}+\left(1-\frac{2 \xi}{h}\right)\left(\nu_{0}-\bar{\nu}\right),\left.\quad \nu_{0} \equiv \nu\right|_{\xi=0}=\chi_{0} \bar{\nu}, \quad \chi_{0} \geq 1, \quad \chi_{0}-1=O(\epsilon),
$$

and the Boussinesq relations $(6.1)_{1--3}$ are taken into account, then (6.3) are satisfied with

$$
\begin{array}{ll}
n_{1}^{s}=\chi_{0}+\left(1-\chi_{0}\right) m_{1}^{s}, & n_{2}^{s}=\chi_{0} m_{2}^{s}+\left(1-\chi_{0}\right) m_{3}^{s}, \\
n_{1}^{f}=\chi_{0}+\left(1-\chi_{0}\right) m_{1}^{f}, & n_{2}^{f}=\chi_{0} m_{2}^{f}+\left(1-\chi_{0}\right) m_{3}^{f} .
\end{array}
$$

Consequently, assuming $(6.1)_{1-3}$, values of $n_{1}^{s}$ to $n_{2}^{f}$ different from those in (6.8), reflect the non-linearity of the solid volume fraction distribution along the avalanche depth.

Finally, by (6.4) of assumption d both pressures $p_{s, f}$ are of the order of the hydrostatic pressure, and the normal extra stresses parallel to the base, i.e., $T_{s, f}^{11}$,

$T_{s, f}^{22}$, as well as the shear stresses $T_{s, f}^{12}, \mathbf{p}_{s, f}$, are small, while the normal extra stresses $T_{s, f}^{33}$ are insignificant. Conditions (6.5), combined with (4.15), show that

$$
\mathbf{p}_{s} \cdot \operatorname{Grad} h+p_{s}-T_{s}^{33}=O\left(\epsilon^{3+\gamma}\right), \quad \mathbf{p}_{f} \cdot \operatorname{Grad} h+p_{f}-T_{f}^{33}=O\left(\epsilon^{3+\gamma}\right)
$$

at $\xi=h(\boldsymbol{\xi}, t)$. These and (6.5) represent nothing more than the assumption that the stress vectors $\boldsymbol{\sigma}_{s, f} \boldsymbol{n}_{F}$ on the free surface are negligibly small, see Luca et al. [11].

In the next section, conditions a- $\mathrm{d}$, complemented by the closure relation for the interaction force $\stackrel{+}{\boldsymbol{m}}$, are used to exploit the equations in (5.5). The body force will be taken as the gravitational force, so that we have, see Luca, Tai and Kuo [10],

$$
\mathbf{b}=-c \mathbf{B}^{-1} \mathbf{s}, \quad \mathrm{b}=-c .
$$

Here non-dimensional components $\mathbf{b}$ and $\mathbf{b}$ of the body force vector $\boldsymbol{b}$ are envisaged.

\section{Quasi-general model for a shallow binary mixture}

It is worthwhile to mention that until now the indices $s$ and $f$ have only been formally used, since the solid-fluid attribute of the mixture has not yet entered the theory. In this section we still keep this generality. However, we specify the interaction force $\stackrel{+}{\boldsymbol{m}}$. Various relations proposed for the interaction force can be found in Massoudi [12]. We also mention Pitman and Le [18], with the law

$$
\stackrel{+}{\boldsymbol{m}}=-\nu \nabla p_{f}+c_{D}(\boldsymbol{u}-\boldsymbol{v}),
$$

where $c_{D}$ is the drag coefficient. Here we take, in a simplified form, the expression of $\underset{\boldsymbol{m}}{\mathbf{r}}$ deduced in Schneider and Hutter [25], see Chapter 8 therein, as a consequence of the entropy inequality within a thermodynamic analysis conducted with specific constitutive assumptions, different from those considered in this paper. It is then clear that (7.1) below is an ad-hoc assumption. However, it has the advantage that it accounts for the influences of both constituent pressures on the interaction force. Besides, an interesting result of this assumption is that, by exploiting the normal 
momentum balance equations, the constituent pressures are shown to be given by the same formulae as for the one-component avalanche mass, see Luca et al. [11].

Thus, introducing the true pressures $\tilde{p}_{s}, \tilde{p}_{f}$ according to

$$
p_{s}=\nu \tilde{p}_{s}, \quad p_{f}=(1-\nu) \tilde{p}_{f},
$$

we assume

$$
\stackrel{+}{\boldsymbol{m}}=\left\{\left(1-c_{s}\right) \tilde{p}_{s}+c_{s} \tilde{p}_{f}\right\} \nabla \nu+c_{D}(\boldsymbol{u}-\boldsymbol{v}),
$$

where $c_{s}$ is the solid mass fraction,

$$
c_{s} \equiv \frac{\rho_{s}}{\rho_{s}+\rho_{f}}=\frac{c_{0} \nu}{c_{0} \nu+1-\nu}, \quad c_{0} \equiv \frac{\tilde{\rho}_{s}}{\tilde{\rho}_{f}}
$$

and the drag coefficient $c_{D}$ is assumed to be constant. In non-dimensional form, (7.1) appears as, see (4.1),

$$
\stackrel{+}{\boldsymbol{m}}=\left\{\left(1-c_{s}\right) \tilde{p}_{s}+\frac{c_{s}}{c_{0}} \tilde{p}_{f}\right\} \nabla \nu+c_{D}(\boldsymbol{u}-\boldsymbol{v}),
$$

where the non-dimensional drag coefficient above is related to the dimensional drag coefficient in (7.1) by

$$
c_{D \text { non-dim }}=\frac{1}{\tilde{\rho}_{s}} \sqrt{\frac{L}{g}} c_{D d i m} .
$$

Moreover, in the curvilinear coordinates considered in section 2, (7.3) emerges as

$$
\begin{gathered}
\stackrel{+}{\mathbf{m}}=\left\{\left(1-c_{s}\right) \tilde{p}_{s}+\frac{c_{s}}{c_{0}} \tilde{p}_{f}\right\} \mathbf{M G r a d} \nu+c_{D}(\mathbf{u}-\mathbf{v}), \\
\stackrel{+}{\mathrm{m}}=\left\{\left(1-c_{s}\right) \tilde{p}_{s}+\frac{c_{s}}{c_{0}} \tilde{p}_{f}\right\} \frac{\partial \nu}{\partial \xi}+c_{D}(\mathrm{u}-\mathrm{v}) .
\end{gathered}
$$

We assume that the non-dimensional drag coefficient in (7.4) is $c_{D}=O(1)^{2}$.

The exploitation of equations (5.5), under assumptions a-d, (6.10), (7.4), leads to the results stated in the next proposition, the proof of which is relegated to the Appendix.

\footnotetext{
${ }^{2}$ In Hydraulics the drag force is defined as the total force acting by a fluid on a submerged rigid body, when the fluid and the rigid body are in relative rectilinear motion. This force is non-dimensionalised as $c_{w} \equiv \operatorname{drag}$ force $/\left((1 / 2) \tilde{\rho}_{f} u^{2} A\right)$, where $u$ is the relative velocity, and $A$ is a typical area of the submerged body. Values of the so-called drag coefficient $c_{w}$ are listed for various bodies, e.g., in Roberson and Crowe [23]: $c_{w} \in(0.85,2.3)$ for large Reynolds numbers, and $c_{w}$ is smaller than these values for laminar flows. Now, if extrapolating, we take the drag force $c_{D \operatorname{dim}}(\boldsymbol{u}-\boldsymbol{v})$ in a binary mixture as $c_{w} \tilde{\rho}_{f}\|\boldsymbol{u}-\boldsymbol{v}\|(\boldsymbol{u}-\boldsymbol{v}) /(2 d)$, where $d$ is a typical diameter of the grain particles, we have $c_{D \text { dim }}=c_{w} \tilde{\rho}_{f}\|\boldsymbol{u}-\boldsymbol{v}\| /(2 d)$. Thus, with the typical values $\tilde{\rho}_{f} \approx 1000 \mathrm{Kg} / \mathrm{m}^{3},\|\boldsymbol{u}-\boldsymbol{v}\| \approx 1 \mathrm{~m} / \mathrm{s}, d=0.1 \mathrm{~m}$ for a debris flow, and taking $c_{w} \in(0.85,2.3)$, we deduce $c_{D \text { dim }} \in\left(4.25 \times 10^{3}, 11.5 \times 10^{3}\right) \mathrm{Kg} /\left(\mathrm{m}^{3} \mathrm{~s}\right)$. Then, with $\tilde{\rho}_{s} \approx 2500 \mathrm{Kg} / \mathrm{m}^{3}, L \approx 100 \mathrm{~m}, g \approx 10 \mathrm{~m} / \mathrm{s}$, we estimate $c_{D \text { non-dim }} \in(5.37,14.54)$. Having in view that $\epsilon \approx 10^{-2}$, we see that the assumption $c_{D \text { non-dim }}=O(1)$ is roughly justified by these heuristics. We also mention Mambretti, Larcan and De Wrachien [13] with their $c_{w}=0.2$, as calibrated in the numerical computations, even for a flow with a large Reynolds number. Using $c_{w}=0.2$, we have $c_{D \text { non-dim }} \approx 1.26=O(1)$. In fact, $c_{D}$ has to be such that $c_{D}(\mathrm{u}-\mathrm{v})=O\left(\epsilon^{\gamma}\right)$, see the derivation of (A.1), (A.2).
} 
Proposition 7.1. Assuming $a-d,(6.10),(7.4)$ and $c_{D}=O(1)$, the mean pressures of the mixture constituents are given by

$$
\bar{p}_{s}=\frac{1}{2} h\left(c+m_{3}^{s} a_{s}\right) \bar{\nu}+O\left(\epsilon^{1+\gamma}\right), \quad \bar{p}_{f}=\frac{1}{2} h\left(c+m_{3}^{f} a_{f}\right)(1-\bar{\nu})+O\left(\epsilon^{1+\gamma}\right),
$$

where $a_{s} \equiv \mathbf{H} \overline{\mathbf{v}} \cdot \overline{\mathbf{v}}, a_{f} \equiv \mathbf{H} \overline{\mathbf{u}} \cdot \overline{\mathbf{u}} . \quad$ Moreover, the depth-integrated mass and tangential momentum balance equations corresponding to the solid constituent emerge as

$$
\begin{gathered}
\frac{\partial}{\partial t}\left\{J_{0} h(1-\Omega h) \bar{\nu}\right\}+\operatorname{Div}\left\{J_{0} h\left(n_{1}^{s}-m_{1}^{s} \Omega h\right) \bar{\nu} \overline{\mathbf{v}}\right\}=O\left(\epsilon^{2+\gamma}\right) \\
\frac{\partial}{\partial t}\left\{J_{0} h\left(n_{1}^{s}-m_{1}^{s} \Omega h\right) \bar{\nu} \overline{\mathbf{v}}\right\}+\operatorname{Div}\left\{J_{0} h\left[\left(n_{2}^{s}-m_{3}^{s} \Omega h\right) \bar{\nu} \overline{\mathbf{v}} \otimes \overline{\mathbf{v}}+\bar{p}_{s} \mathbf{M}_{0}-\overline{\mathbf{P}}_{s}\right]\right\} \\
-J_{0} h\left\{\mathbf{F}^{-1} \frac{\partial \mathbf{F}}{\partial \xi^{\alpha}} \overline{\mathbf{P}}_{s} \mathbf{e}_{\alpha}-2 \mathbf{W} \overline{\mathbf{p}}_{s}+\left(\mathbf{H} \cdot \overline{\mathbf{P}}_{s}\right) \mathbf{F}^{-1} \mathbf{s}\right\} \\
=-\left.J_{0} \mathbf{p}_{s}\right|_{\xi=0}-J_{0} h\left\{\left(c+n_{2}^{s} a_{s}-\frac{1}{2} m_{3}^{s} h \tilde{a}_{s}\right) \bar{\nu} \mathbf{I}+\bar{p}_{s} \mathbf{W}\right\} \mathbf{F}^{-1} \mathbf{s} \\
-\mathbf{F}^{-1} \frac{\partial \mathbf{F}}{\partial \xi^{\alpha}}\left\{J_{0} h\left[\left(n_{2}^{s}-m_{3}^{s} \Omega h\right) \bar{\nu} \overline{\mathbf{v}} \otimes \overline{\mathbf{v}}+\bar{p}_{s} \mathbf{M}_{0}\right]\right\} \mathbf{e}_{\alpha} \\
+\frac{1}{2} J_{0} h^{2} m_{3}^{s} \bar{\nu} \mathbf{F}-1 \frac{\partial}{\partial \xi^{\alpha}}(\mathbf{F} \mathbf{W} \mathbf{F}-1) \mathbf{F}\left(\overline{\mathbf{v}} \otimes \overline{\mathbf{v}}^{-\mathbf{e}_{\alpha}}+J_{0} h^{2} \beta_{s} \bar{\nu} \mathbf{W} \overline{\mathbf{v}}\right. \\
+\frac{J_{0} h^{2}}{2\left(c_{0} \bar{\nu}+1-\bar{\nu}\right)}\left\{c+m_{3}^{s}(1-\bar{\nu}) a_{s}+m_{3}^{f} \bar{\nu} a_{f}\right\} \mathbf{M}_{0} \mathrm{Grad} \bar{\nu} \\
+c_{D} J_{0} h\left\{\left(1-m_{1}^{f} \Omega h\right) \overline{\mathbf{u}}-\left(1-m_{1}^{s} \Omega h\right) \overline{\mathbf{v}}\right\}+O\left(\epsilon^{2+\gamma}\right),
\end{gathered}
$$

where $\tilde{a}_{s} \equiv \mathbf{H} \overline{\mathbf{v}} \cdot \mathbf{W} \overline{\mathbf{v}}$. Similarly, the depth-integrated mass and tangential momentum balance equations corresponding to the fluid constituent turn into

$$
\begin{aligned}
\frac{\partial}{\partial t}\left\{J_{0}\right. & h(1-\Omega h)(1-\bar{\nu})\}+\operatorname{Div}\left\{J_{0} h\left(1-n_{1}^{f} \bar{\nu}-m_{1}^{f}(1-\bar{\nu}) \Omega h\right) \overline{\mathbf{u}}\right\}=O\left(\epsilon^{2+\gamma}\right), \\
\frac{\partial}{\partial t}\{ & \left.J_{0} h\left(1-n_{1}^{f} \bar{\nu}-m_{1}^{f}(1-\bar{\nu}) \Omega h\right) \overline{\mathbf{u}}\right\} \\
& +\operatorname{Div}\left\{J_{0} h\left[\left(m_{2}^{f}-n_{2}^{f} \bar{\nu}-m_{3}^{f}(1-\bar{\nu}) \Omega h\right) \overline{\mathbf{u}} \otimes \overline{\mathbf{u}}+\bar{p}_{f} \mathbf{M}_{0}-\overline{\mathbf{P}}_{f}\right]\right\} \\
& -J_{0} h\left\{\mathbf{F}^{-1} \frac{\partial \mathbf{F}}{\partial \xi^{\alpha}} \overline{\mathbf{P}}_{f} \mathbf{e}_{\alpha}-2 \mathbf{W} \overline{\mathbf{p}}_{f}+\left(\mathbf{H} \cdot \overline{\mathbf{P}}_{f}\right) \mathbf{F}^{-1} \mathbf{s}\right\}=-\left.J_{0} \mathbf{p}_{f}\right|_{\xi=0} \\
& -J_{0} h\left\{\left[\left(m_{2}^{f}-n_{2}^{f} \bar{\nu}\right) a_{f}+\left(c-\frac{1}{2} m_{3}^{f} h \tilde{a}_{f}\right)(1-\bar{\nu})\right] \mathbf{I}+\bar{p}_{f} \mathbf{W}\right\} \mathbf{F}^{-1} \mathbf{s} \\
& -\mathbf{F}^{-1} \frac{\partial \mathbf{F}}{\partial \xi^{\alpha}}\left\{J_{0} h\left[\left(m_{2}^{f}-n_{2}^{f} \bar{\nu}-m_{3}^{f}(1-\bar{\nu}) \Omega h\right) \overline{\mathbf{u}} \otimes \overline{\mathbf{u}}+\bar{p}_{f} \mathbf{M}_{0}\right]\right\} \mathbf{e}_{\alpha} \\
& +\frac{1}{2} J_{0} h^{2} m_{3}^{f}(1-\bar{\nu}) \mathbf{F}^{-1} \frac{\partial}{\partial \xi^{\alpha}}\left(\mathbf{F} \mathbf{W} \mathbf{F}^{-1}\right) \mathbf{F}(\overline{\mathbf{u}} \otimes \overline{\mathbf{u}}) \mathbf{e}_{\alpha}+J_{0} h^{2} \beta_{f}(1-\bar{\nu}) \mathbf{W} \overline{\mathbf{u}} \\
& -\frac{c_{0} J_{0} h^{2}}{2\left(c_{0} \bar{\nu}+1-\bar{\nu}\right)}\left\{c+m_{3}^{s}(1-\bar{\nu}) a_{s}+m_{3}^{f} \bar{\nu} a_{f}\right\} \mathbf{M}_{0} \mathrm{Grad} \bar{\nu} \\
& -c_{0} c_{D} J_{0} h\left\{\left(1-m_{1}^{f} \Omega h\right) \overline{\mathbf{u}}-\left(1-m_{1}^{s} \Omega h\right) \overline{\mathbf{v}}\right\}+O\left(\epsilon^{2+\gamma}\right),
\end{aligned}
$$

where $\tilde{a}_{f} \equiv \mathbf{H} \overline{\mathbf{u}} \cdot \mathbf{W} \overline{\mathbf{u}}$. 
In $(7.7),(7.9)$ the mean pressures $\bar{p}_{s}, \bar{p}_{f}$ are understood to be given by the nonneglected parts in (7.5). It is worthwhile to note that the formulae (A.6) (see Appendix) for the true pressures of both constituents coincide with the formula for the pressure in the one-component theory, see Luca et al. [11]. This result is essentially due to assumption (7.3) on the interaction force plus the ordering approximations a-d.

We refer to (7.5)-(7.9) as the shallow avalanche equations corresponding to a two-component mixture, for which the non-dimensional interaction force $\stackrel{+}{\boldsymbol{m}}$ is $(7.3)$. Complemented by closure relations for the extra stresses of both constituents, these equations are meant to stand for the determination of $\bar{\nu}, \overline{\mathbf{v}}, \overline{\mathbf{u}}, h$.

Multiplication of (7.7) from the left by $\mathbf{F}$ gives the equation

$$
\begin{array}{rl}
\frac{\partial}{\partial t}\{ & \left.J_{0} h\left(n_{1}^{s}-m_{1}^{s} \Omega h\right) \bar{\nu} \mathbf{F} \overline{\mathbf{v}}\right\} \\
& +\operatorname{Div}\left\{J_{0} h \mathbf{F}\left[\left(n_{2}^{s}-m_{3}^{s} \Omega h\right) \bar{\nu} \overline{\mathbf{v}} \otimes \overline{\mathbf{v}}+\bar{p}_{s} \mathbf{M}_{0}-\overline{\mathbf{P}}_{s}\right]\right\} \\
& +J_{0} h\left\{2 \mathbf{F} \mathbf{W} \overline{\mathbf{p}}_{s}-\left(\mathbf{H} \cdot \overline{\mathbf{P}}_{s}\right) \mathbf{s}\right\} \\
=-J_{0} & \left.\mathbf{F} \mathbf{p}_{s}\right|_{\xi=0}-J_{0} h\left\{\left(c+n_{2}^{s} a_{s}-\frac{1}{2} m_{3}^{s} h \tilde{a}_{s}\right) \bar{\nu} \mathbf{I}+\bar{p}_{s} \mathbf{F} \mathbf{W} \mathbf{F}^{-1}\right\} \mathbf{s} \\
& -\frac{1}{2} J_{0} h^{2} m_{3}^{s} \bar{\nu} \frac{\partial}{\partial \xi^{\alpha}}\left(\mathbf{F W} \mathbf{F}^{-1}\right) \mathbf{F}(\overline{\mathbf{v}} \otimes \overline{\mathbf{v}}) \mathbf{e}_{\alpha}+J_{0} h^{2} \beta_{s} \bar{\nu} \mathbf{F W} \overline{\mathbf{v}} \\
& +\frac{J_{0} h^{2}}{2\left(c_{0} \bar{\nu}+1-\bar{\nu}\right)}\left\{c+m_{3}^{s}(1-\bar{\nu}) a_{s}+m_{3}^{f} \bar{\nu} a_{f}\right\} \mathbf{F} \mathbf{M}_{0} \operatorname{Grad} \bar{\nu} \\
& +c_{D} J_{0} h\left\{\left(1-m_{1}^{f} \Omega h\right) \mathbf{F} \overline{\mathbf{u}}-\left(1-m_{1}^{s} \Omega h\right) \mathbf{F} \overline{\mathbf{v}}\right\}+O\left(\epsilon^{2+\gamma}\right),
\end{array}
$$

which is equivalent to (7.7), since $\operatorname{det} \mathbf{F} \neq 0$, and somewhat of lower complexity than that of (7.7). A similar equation can be deduced for the fluid constituent from (7.9), namely

$$
\begin{aligned}
& \frac{\partial}{\partial t}\{\left.J_{0} h\left(1-n_{1}^{f} \bar{\nu}-m_{1}^{f}(1-\bar{\nu}) \Omega h\right) \mathbf{F} \overline{\mathbf{u}}\right\} \\
&+\operatorname{Div}\left\{J_{0} h \mathbf{F}\left[\left(m_{2}^{f}-n_{2}^{f} \bar{\nu}-m_{3}^{f}(1-\bar{\nu}) \Omega h\right) \overline{\mathbf{u}} \otimes \overline{\mathbf{u}}+\bar{p}_{f} \mathbf{M}_{0}-\overline{\mathbf{P}}_{f}\right]\right\} \\
&+J_{0} h\left\{2 \mathbf{F W} \overline{\mathbf{p}}_{f}-\left(\mathbf{H} \cdot \overline{\mathbf{P}}_{f}\right) \mathbf{s}\right\} \\
&=-\left.J_{0} \mathbf{F} \mathbf{p}_{f}\right|_{\xi=0}-J_{0} h\left\{\left[\left(m_{2}^{f}-n_{2}^{f} \bar{\nu}\right) a_{f}+\left(c-\frac{1}{2} m_{3}^{f} h \tilde{a}_{f}\right)(1-\bar{\nu})\right] \mathbf{I}+\bar{p}_{f} \mathbf{F} \mathbf{W} \mathbf{F}^{-1}\right\} \mathbf{s} \\
& \quad+\frac{1}{2} J_{0} h^{2} m_{3}^{f}(1-\bar{\nu}) \frac{\partial}{\partial \xi^{\alpha}}\left(\mathbf{F} \mathbf{W} \mathbf{F}^{-1}\right) \mathbf{F}(\overline{\mathbf{u}} \otimes \overline{\mathbf{u}}) \mathbf{e}_{\alpha}+J_{0} h^{2} \beta_{f}(1-\bar{\nu}) \mathbf{F W} \overline{\mathbf{u}} \\
& \quad-\frac{c_{0} J_{0} h^{2}}{2\left(c_{0} \bar{\nu}+1-\bar{\nu}\right)}\left\{c+m_{3}^{s}(1-\bar{\nu}) a_{s}+m_{3}^{f} \bar{\nu} a_{f}\right\} \mathbf{F} \mathbf{M}_{0} \operatorname{Grad} \bar{\nu} \\
& \quad-c_{0} c_{D} J_{0} h\left\{\left(1-m_{1}^{f} \Omega h\right) \mathbf{F} \overline{\mathbf{u}}-\left(1-m_{1}^{s} \Omega h\right) \mathbf{F} \overline{\mathbf{v}}\right\}+O\left(\epsilon^{2+\gamma}\right) .
\end{aligned}
$$

For simplicity of writing, in the remainder of the paper we shall refer to (7.10) and (7.11) and not to (7.7), (7.9).

The mean pressures (7.5) and equations (7.6), (7.10), (7.8) and (7.11) significantly simplify if the bottom topography is slightly curved, as shown in the next proposition. So, we have 
Proposition 7.2. If $\mathcal{H}=O(\epsilon)$, or $\mathcal{H}=O\left(\epsilon^{\gamma}\right)$ and $\mathbf{p}_{s}=O(\epsilon)$, $\mathbf{p}_{f}=O(\epsilon)$, then the mean pressures of the mixture constituents are given by

$$
\bar{p}_{s}=\frac{1}{2} h c \bar{\nu}+O\left(\epsilon^{1+\gamma}\right), \quad \bar{p}_{f}=\frac{1}{2} h c(1-\bar{\nu})+O\left(\epsilon^{1+\gamma}\right),
$$

the depth-integrated mass and tangential momentum balance equations corresponding to the solid constituent emerge as

$$
\begin{gathered}
\frac{\partial}{\partial t}\left\{J_{0} h \bar{\nu}\right\}+\operatorname{Div}\left\{J_{0} h n_{1}^{s} \bar{\nu} \overline{\mathbf{v}}\right\}=O\left(\epsilon^{2+\gamma}\right), \\
\frac{\partial}{\partial t}\left\{J_{0} h n_{1}^{s} \bar{\nu} \mathbf{F} \overline{\mathbf{v}}\right\}+\operatorname{Div}\left\{J_{0} h \mathbf{F}\left[n_{2}^{s} \bar{\nu} \overline{\mathbf{v}} \otimes \overline{\mathbf{v}}+\bar{p}_{s} \mathbf{M}_{0}-\overline{\mathbf{P}}_{s}\right]\right\} \\
=-\left.J_{0} \mathbf{F} \mathbf{p}_{s}\right|_{\xi=0}-J_{0} h\left(c+n_{2}^{s} a_{s}\right) \bar{\nu} \mathbf{s}+\frac{c J_{0} h^{2}}{2\left(c_{0} \bar{\nu}+1-\bar{\nu}\right)} \mathbf{F M}_{0} \operatorname{Grad} \bar{\nu} \\
+c_{D} J_{0} h(\mathbf{F} \overline{\mathbf{u}}-\mathbf{F} \overline{\mathbf{v}})+O\left(\epsilon^{2+\gamma}\right),
\end{gathered}
$$

and the depth-integrated mass and tangential momentum balance equations corresponding to the fluid constituent turn into

$$
\begin{gathered}
\frac{\partial}{\partial t}\left\{J_{0} h(1-\bar{\nu})\right\}+\operatorname{Div}\left\{J_{0} h\left(1-n_{1}^{f} \bar{\nu}\right) \overline{\mathbf{u}}\right\}=O\left(\epsilon^{2+\gamma}\right), \\
\frac{\partial}{\partial t}\left\{J_{0} h\left(1-n_{1}^{f} \bar{\nu}\right) \mathbf{F} \overline{\mathbf{u}}\right\}+\operatorname{Div}\left\{J_{0} h \mathbf{F}\left[\left(m_{2}^{f}-n_{2}^{f} \bar{\nu}\right) \overline{\mathbf{u}} \otimes \overline{\mathbf{u}}+\bar{p}_{f} \mathbf{M}_{0}-\overline{\mathbf{P}}_{f}\right]\right\} \\
=-\left.J_{0} \mathbf{F} \mathbf{p}_{f}\right|_{\xi=0}-J_{0} h\left\{\left(m_{2}^{f}-n_{2}^{f} \bar{\nu}\right) a_{f}+c(1-\bar{\nu})\right\} \mathbf{s} \\
-\frac{c_{0} c J_{0} h^{2}}{2\left(c_{0} \bar{\nu}+1-\bar{\nu}\right)} \mathbf{F M}_{0} \operatorname{Grad} \bar{\nu}-c_{0} c_{D} J_{0} h(\mathbf{F} \overline{\mathbf{u}}-\mathbf{F} \overline{\mathbf{v}})+O\left(\epsilon^{2+\gamma}\right) .
\end{gathered}
$$

\section{Particular models for a shallow solid-fluid mixture}

In this section we "close" the balance equations (7.6), (7.10), (7.8) and (7.11) by specifying the extra-stresses

$$
\overline{\mathbf{P}}_{s}, \quad \overline{\mathbf{P}}_{f}, \quad \overline{\mathbf{p}}_{s}, \quad \overline{\mathbf{p}}_{f},\left.\quad \mathbf{p}_{s}\right|_{\xi=0},\left.\quad \mathbf{p}_{f}\right|_{\xi=0} .
$$

We present three avalanche models of binary mixtures on arbitrary topography, by combining one fluid constitutive behaviour with three different solid constitutive behaviours.

Consider first the interstitial incompressible fluid. For this we assume the (nondimensional) Newtonian/non-Newtonian law

$$
\boldsymbol{\sigma}_{f}=-p_{f} \boldsymbol{1}+2 \eta(\dot{\gamma}) \boldsymbol{D}, \quad \dot{\gamma} \equiv 2 \sqrt{I I_{\boldsymbol{D}}}, \quad I I_{\boldsymbol{D}} \equiv \frac{1}{2} \operatorname{tr} \boldsymbol{D}^{2}, \quad \eta=O\left(\epsilon^{2+\gamma}\right),
$$

with $p_{f}$ - the pore fluid pressure, $\mathbf{1}$ - the unit tensor, $\boldsymbol{D}$ - the stretching tensor corresponding to the fluid constituent,

$$
\boldsymbol{D} \equiv \frac{1}{2}\left(\nabla \boldsymbol{u}+(\nabla \boldsymbol{u})^{T}\right),
$$

and $\eta$ - the dynamic viscosity. We note that, according to the scalings (4.1), a fluid with non-dimensional dynamic viscosity of order $O\left(\epsilon^{2+\gamma}\right)$ is a realistic model, see Luca 
et al. [11]. We identify $p_{f}, \boldsymbol{\sigma}_{f}^{E}$ in (3.11) with $p_{f}$ and $2 \eta(\dot{\gamma}) \boldsymbol{D}$, respectively, from (8.1). Moreover, we suppose that at the bottom topography there is friction, modeled by the (dimensional) viscous law

$$
\boldsymbol{\sigma}_{f} \boldsymbol{n}-\left(\boldsymbol{\sigma}_{f} \boldsymbol{n} \cdot \boldsymbol{n}\right) \boldsymbol{n}=\rho_{f} \tilde{c}\|\boldsymbol{u}\| \boldsymbol{u} \quad \text { at } \quad \xi=0,
$$

with (non-dimensional) constant friction coefficient $\tilde{c}$. In non-dimensional form this law emerges as

$$
\boldsymbol{\sigma}_{f} \boldsymbol{n}-\left(\boldsymbol{\sigma}_{f} \boldsymbol{n} \cdot \boldsymbol{n}\right) \boldsymbol{n}=(1-\nu) \tilde{c}\|\boldsymbol{u}\| \boldsymbol{u} \quad \text { at } \quad \xi=0 .
$$

Since now

$$
\mathbf{P}_{f}=O(\eta)=O\left(\epsilon^{2+\gamma}\right), \quad \mathbf{p}_{f}=O(\eta / \epsilon)=O\left(\epsilon^{1+\gamma}\right), \quad T_{f}^{33}=O(\eta)=O\left(\epsilon^{2+\gamma}\right),
$$

(see the contravariant components of the stretching tensor with respect to the basis $\left\{\boldsymbol{g}_{1}, \boldsymbol{g}_{2}, \boldsymbol{g}_{3}\right\}$ in Luca et al. [10] or [11] and use (8.1)), the dynamic approximations (6.4) referring to $\mathbf{P}_{f}, \mathbf{p}_{f}, T_{f}^{33}$ are fulfilled, conditions $(6.5)_{2},(6.9)_{2}$ at the free surface make sense, and the only non-negligible stress term in (7.11) is $\left.\mathbf{p}_{f}\right|_{\xi=0}$. This term is inferred from the boundary condition (8.2). Indeed, in curvilinear coordinates (2.3) this condition emerges as

$$
\left.\mathbf{p}_{f}\right|_{\xi=0}=\left(1-\nu_{0}\right) \tilde{c} \sqrt{\mathbf{M}_{0}^{-1} \mathbf{u}_{0} \cdot \mathbf{u}_{0}} \mathbf{u}_{0},\left.\quad \mathbf{u}_{0} \equiv \mathbf{u}\right|_{\xi=0} .
$$

Since $\mathbf{p}_{f}=O\left(\epsilon^{1+\gamma}\right)$ in the bulk fluid, we require $\tilde{c}=O\left(\epsilon^{1+\gamma}\right)$, so as to have $\left.\mathbf{p}_{f}\right|_{\xi=0}$ as given by (8.3) of order $O\left(\epsilon^{1+\gamma}\right)$. Then, in order to relate the basal shear stress (8.3) to $\bar{\nu}$ and $\overline{\mathbf{u}}$, we note that $\nu_{0}=\bar{\nu}+O(\epsilon)$, see (6.2), and assume

$$
\mathbf{u}_{0}=\chi_{f} \overline{\mathbf{u}}, \quad \chi_{f} \in(0,1],
$$

which yields

$$
\left.\mathbf{p}_{f}\right|_{\xi=0}=(1-\bar{\nu}) \tilde{c} \chi_{f}^{2} \sqrt{\mathbf{M}_{0}^{-1} \overline{\mathbf{u}} \cdot \overline{\mathbf{u}}} \overline{\mathbf{u}}+O\left(\epsilon^{2+\gamma}\right)
$$

Generally, the sliding parameter $\chi_{f}$ must be prescribed. For the power law velocity profile

$$
\mathbf{u}=\overline{\mathbf{u}}+\frac{1}{n+1}\left\{1-(n+2)\left(1-\frac{\xi}{h}\right)^{n+1}\right\}\left(\overline{\mathbf{u}}-\mathbf{u}_{0}\right), \quad n>0,
$$

and for a constant viscosity $\eta$, following Luca, Tai and Kuo [10] it can be shown that, equating $\left.\mathbf{p}_{f}\right|_{\xi=0}$ as given by the constitutive law (in which the power law velocity profile is accounted for) and $\left.\mathbf{p}_{f}\right|_{\xi=0}$ as given by the friction law, see (8.3), one obtains

$$
\mathbf{u}_{0}=\chi_{f} \overline{\mathbf{u}}+O\left(\epsilon^{2+\gamma}\right), \quad \chi_{f}=2\left\{1+\sqrt{1+4 \tilde{m} \sqrt{\mathbf{M}_{0}^{-1} \overline{\mathbf{u}} \cdot \overline{\mathbf{u}}}}\right\}^{-1}, \quad \tilde{m} \equiv \frac{(1-\bar{\nu}) \tilde{c} h}{\eta(n+2)},
$$

which clearly shows that the sliding coefficient depends on $\eta, \tilde{c}$, and hence $\chi_{f}$ is a parameter which depends, in particular, on the rheology of the fluid and on the friction properties at the bed surface. 
Thus, postulating the Newtonian law (8.1) with $\eta=O\left(\epsilon^{2+\gamma}\right)$, and the viscous friction law (8.2) with $\tilde{c}=O\left(\epsilon^{1+\gamma}\right)$, the tangential momentum balance equation (7.11) corresponding to the fluid constituent emerges as

$$
\begin{aligned}
\frac{\partial}{\partial t}\{ & \left.J_{0} h\left(1-n_{1}^{f} \bar{\nu}-m_{1}^{f}(1-\bar{\nu}) \Omega h\right) \mathbf{F} \overline{\mathbf{u}}\right\} \\
& +\operatorname{Div}\left\{J_{0} h \mathbf{F}\left[\left(m_{2}^{f}-n_{2}^{f} \bar{\nu}-m_{3}^{f}(1-\bar{\nu}) \Omega h\right) \overline{\mathbf{u}} \otimes \overline{\mathbf{u}}+\bar{p}_{f} \mathbf{M}_{0}\right]\right\} \\
=-J_{0}(1-\bar{\nu}) \tilde{c} \chi_{f}^{2} \sqrt{\mathbf{M}_{0}^{-1} \overline{\mathbf{u}} \cdot \overline{\mathbf{u}} \mathbf{F} \overline{\mathbf{u}}} & \\
& -J_{0} h\left\{\left[\left(m_{2}^{f}-n_{2}^{f} \bar{\nu}\right) a_{f}+\left(c-\frac{1}{2} m_{3}^{f} h \tilde{a}_{f}\right)(1-\bar{\nu})\right] \mathbf{I}+\bar{p}_{f} \mathbf{F} \mathbf{W} \mathbf{F}^{-1}\right\} \mathbf{s} \\
& +\frac{1}{2} J_{0} h^{2} m_{3}^{f}(1-\bar{\nu}) \frac{\partial}{\partial \xi^{\alpha}}\left(\mathbf{F} \mathbf{W} \mathbf{F}^{-1}\right) \mathbf{F}(\overline{\mathbf{u}} \otimes \overline{\mathbf{u}}) \mathbf{e}_{\alpha}+J_{0} h^{2} \beta_{f}(1-\bar{\nu}) \mathbf{F W} \overline{\mathbf{u}} \\
& -\frac{c_{0} J_{0} h^{2}}{2\left(c_{0} \bar{\nu}+1-\bar{\nu}\right)}\left\{c+m_{3}^{s}(1-\bar{\nu}) a_{s}+m_{3}^{f} \bar{\nu} a_{f}\right\} \mathbf{F} \mathbf{M}_{0} \operatorname{Grad}_{\bar{\nu}} \\
& -c_{0} c_{D} J_{0} h\left\{\left(1-m_{1}^{f} \Omega h\right) \mathbf{F} \overline{\mathbf{u}}-\left(1-m_{1}^{s} \Omega h\right) \mathbf{F} \overline{\mathbf{v}}\right\}+O\left(\epsilon^{2+\gamma}\right) .
\end{aligned}
$$

For small curvature of the bed surface, i.e., $\mathcal{H}=O\left(\epsilon^{\gamma}\right)$, the equation above reduces to

$$
\begin{array}{r}
\frac{\partial}{\partial t}\left\{J_{0} h\left(1-n_{1}^{f} \bar{\nu}\right) \mathbf{F} \overline{\mathbf{u}}\right\}+\operatorname{Div}\left\{J_{0} h \mathbf{F}\left[\left(m_{2}^{f}-n_{2}^{f} \bar{\nu}\right) \overline{\mathbf{u}} \otimes \overline{\mathbf{u}}+\bar{p}_{f} \mathbf{M}_{0}\right]\right\} \\
=-J_{0}(1-\bar{\nu}) \tilde{c} \chi_{f}^{2} \sqrt{\mathbf{M}_{0}^{-1} \overline{\mathbf{u}} \cdot \overline{\mathbf{u}}} \mathbf{F} \overline{\mathbf{u}}-J_{0} h\left\{\left(m_{2}^{f}-n_{2}^{f} \bar{\nu}\right) a_{f}+c(1-\bar{\nu})\right\} \mathbf{s} \\
-\frac{c_{0} c J_{0} h^{2}}{2\left(c_{0} \bar{\nu}+1-\bar{\nu}\right)} \mathbf{F} \mathbf{M}_{0} \operatorname{Grad} \bar{\nu}-c_{0} c_{D} J_{0} h(\mathbf{F} \overline{\mathbf{u}}-\mathbf{F} \overline{\mathbf{v}})+O\left(\epsilon^{2+\gamma}\right),
\end{array}
$$

see also $(7.16)$.

Now we refer to the solid constituent, for which we identify the pressure $p_{s}$ in decomposition (3.11) as $p_{s} \equiv-\boldsymbol{\sigma}_{s} \boldsymbol{n} \cdot \boldsymbol{n}$, so that $T_{s}^{33}=0$. At the bottom topography we assume the classical Coulomb friction law

$$
\boldsymbol{\sigma}_{s} \boldsymbol{n}-\left(\boldsymbol{\sigma}_{s} \boldsymbol{n} \cdot \boldsymbol{n}\right) \boldsymbol{n}=(\tan \delta)\left(-\boldsymbol{\sigma}_{s} \boldsymbol{n} \cdot \boldsymbol{n}\right)_{+} \operatorname{sgn} \boldsymbol{v} \quad \text { at } \xi=0,
$$

with $\tan \delta=O\left(\epsilon^{\gamma}\right)$, where $\delta$ is the basal angle of friction, $\tan \delta>0$, the index + stands for the positive part of a quantity, i.e., $f_{+} \equiv \max \{0, f\}$, and

$$
\operatorname{sgn} \boldsymbol{v} \equiv\left\{\begin{array}{l}
\frac{1}{\|\boldsymbol{v}\|} \boldsymbol{v}, \quad \text { if } \boldsymbol{v} \neq \boldsymbol{O}, \\
\text { any tangent vector } \boldsymbol{m} \text { to } \mathcal{S},\|\boldsymbol{m}\| \leq 1, \quad \text { if } \boldsymbol{v}=\boldsymbol{O},
\end{array}\right.
$$

see, e.g., Bouchut and Westdickenberg [1]. In (8.5) the non-dimensional form according to (4.1) is envisaged. Recalling that $\mathrm{v}=0$ at $\xi=0$, in curvilinear coordinates (8.5) emerges as

$$
\left.\mathbf{p}_{s}\right|_{\xi=0}=(\tan \delta)\left(\left.p_{s}\right|_{\xi=0}\right)_{+} \operatorname{sgn} \mathbf{v}_{0},\left.\quad \mathbf{v}_{0} \equiv \mathbf{v}\right|_{\xi=0},
$$

where for a 2-column $\mathbf{x}$ the multivalued function $\operatorname{sgn} \mathbf{x}$ is defined as

$$
\operatorname{sgn} \mathbf{x} \equiv\left\{\begin{array}{l}
\frac{1}{\sqrt{\mathbf{M}_{0}^{-1} \mathbf{x} \cdot \mathbf{x}}} \mathbf{x}, \quad \text { if } \mathbf{x} \neq \mathbf{0}, \\
\text { any 2-column } \mathbf{m}, \mathbf{M}_{0}^{-1} \mathbf{m} \cdot \mathbf{m} \leq 1, \quad \text { if } \mathbf{x}=\mathbf{0} .
\end{array} .\right.
$$


In particular, (8.6) shows that $\left.\mathbf{p}_{s}\right|_{\xi=0}=O\left(\epsilon^{1+\gamma}\right)$. We assume that $\mathbf{p}_{s}$ in the bulk fluid is of the same order of magnitude as on the bottom surface, that is,

$$
\mathbf{p}_{s}=O\left(\epsilon^{1+\gamma}\right) \text {. }
$$

As a consequence, in (7.10) the term $2 J_{0} h \mathbf{W} \overline{\mathbf{p}}_{s}$ is negligibly small. Moreover, with $\mathbf{p}_{s}=O\left(\epsilon^{1+\gamma}\right)$ and $T_{s}^{33}=0$, we have

$$
p_{s}=\bar{\nu} \tilde{p}_{s}^{\star}+O\left(\epsilon^{2}\right), \quad \tilde{p}_{s}^{\star} \equiv c(h-\xi)+\mathbf{H} \cdot \int_{\xi}^{h(\boldsymbol{\xi}, t)} \mathbf{v} \otimes \mathbf{v} d \xi^{\prime},
$$

see (A.7) and the derivation of (A.6). So, taking $\xi=0$ in the expression above, we derive

$$
\left.p_{s}\right|_{\xi=0}=h\left(c+m_{2}^{s} a_{s}\right) \bar{\nu}+O\left(\epsilon^{2}\right) .
$$

This result and the assumption

$$
\mathbf{v}_{0}=\chi_{s} \overline{\mathbf{v}}, \quad \chi_{s} \in(0,1]
$$

turn the boundary condition (8.6) into

$$
\left.\mathbf{p}_{s}\right|_{\xi=0}=h(\tan \delta)\left\{c+m_{2}^{s} a_{s}\right\}_{+} \bar{\nu} \operatorname{sgn} \overline{\mathbf{v}}+O\left(\epsilon^{2+\gamma}\right),
$$

which needs to be used in (7.10) to express the basal shear stress $\left.\mathbf{p}_{s}\right|_{\xi=0}$. The sliding coefficient $\chi_{s}$ does not appear in the friction law (8.7).

It only remains to specify the mean extra stresses $\overline{\mathbf{P}}_{s}$. To this end, recalling the assumption $\mathbf{P}_{s}=O(\epsilon)$, we shall specify $\overline{\boldsymbol{\sigma}}_{s}$ when evaluated up to terms of order $O\left(\epsilon^{1+\gamma}\right)$. More specifically, we have

$$
\boldsymbol{\sigma}_{s}=\sigma_{s}^{i j} \boldsymbol{g}_{i} \otimes \boldsymbol{g}_{j}=\sigma_{s}^{\alpha \beta} \boldsymbol{g}_{\alpha} \otimes \boldsymbol{g}_{\beta}+\sigma_{s}^{\alpha 3}\left(\boldsymbol{g}_{\alpha} \otimes \boldsymbol{n}+\boldsymbol{n} \otimes \boldsymbol{g}_{\alpha}\right)+\sigma_{s}^{33} \boldsymbol{n} \otimes \boldsymbol{n},
$$

and using the relation

$$
\left(\sigma_{s}^{i j}\right)=\left(\begin{array}{cc}
-p_{s} \mathbf{M}+\mathbf{P}_{s} & \mathbf{p}_{s} \\
\mathbf{p}_{s}^{T} & -p_{s}+T_{s}^{33}
\end{array}\right)
$$

between the contravariant components $\sigma_{s}^{i j}$ of the stress tensor $\boldsymbol{\sigma}_{s}$ and the contravariant components $\mathbf{P}_{s}, \mathbf{p}_{s}, T_{s}^{33}$ of the extra stress tensor $\boldsymbol{\sigma}_{s}^{E}$ (see (2.10) and (3.11) $)_{1}$ ), as well as the assumptions

$$
\mathbf{P}_{s}=O(\epsilon), \quad \mathbf{p}_{s}=O\left(\epsilon^{1+\gamma}\right), \quad p_{s}=-\sigma_{s}^{33}=O(\epsilon)
$$

and approximation $\boldsymbol{g}_{\alpha}=\boldsymbol{\tau}_{\alpha}+O(\epsilon)$, see (2.7), we have

$$
\boldsymbol{\sigma}_{s}=\sigma_{s}^{\star \alpha \beta} \boldsymbol{\tau}_{\alpha} \otimes \boldsymbol{\tau}_{\beta}-p_{s} \boldsymbol{n} \otimes \boldsymbol{n}+O\left(\epsilon^{1+\gamma}\right)=O(\epsilon), \quad\left(\sigma_{s}^{\star \alpha \beta}\right) \equiv-p_{s} \mathbf{M}_{0}+\mathbf{P}_{s} .
$$

This yields

$$
\begin{gathered}
\overline{\boldsymbol{\sigma}}_{s}=\overline{\boldsymbol{\sigma}}_{s}^{\star}+O\left(\epsilon^{1+\gamma}\right), \\
\overline{\boldsymbol{\sigma}}_{s}^{\star} \equiv \bar{\sigma}_{s}^{\star \alpha \beta} \boldsymbol{\tau}_{\alpha} \otimes \boldsymbol{\tau}_{\beta}-\bar{p}_{s} \boldsymbol{n} \otimes \boldsymbol{n}, \quad\left(\bar{\sigma}_{s}^{\star \alpha \beta}\right)=-\bar{p}_{s} \mathbf{M}_{0}+\overline{\mathbf{P}}_{s},
\end{gathered}
$$

and therefore it is clear that any assumption on $\overline{\boldsymbol{\sigma}}_{s}$ as evaluated up to terms $O\left(\epsilon^{1+\gamma}\right)$, that is on $\overline{\boldsymbol{\sigma}}_{s}^{\star}$, yields $\overline{\mathbf{P}}_{s}$. Moreover, (8.8) shows that $\overline{\boldsymbol{\sigma}}_{s}^{\star} \boldsymbol{n}=-\bar{p}_{s} \boldsymbol{n}$, implying that $-\bar{p}_{s}$ 
is an eigenvalue of $\overline{\boldsymbol{\sigma}}_{s}^{\star}$, and $\boldsymbol{n}$ is an eigenvector corresponding to this eigenvalue. As a symmetric tensor, $\overline{\boldsymbol{\sigma}}_{s}^{\star}$ is then completely determined if the other two eigenvalues and principal directions (mutually orthogonal and perpendicular to $\boldsymbol{n}$ ) are specified. We shall give them in terms of the eigenvalues and eigenvectors of the mean surface stretching corresponding to the solid constituent, that is of $\boldsymbol{D}_{\mathcal{S}} \overline{\boldsymbol{v}}_{\tau}$,

$$
\boldsymbol{D}_{\mathcal{S}} \overline{\boldsymbol{v}}_{\tau} \equiv \frac{1}{2}\left(\nabla_{\mathcal{S}} \overline{\boldsymbol{v}}_{\tau}+\left(\nabla_{\mathcal{S}} \overline{\boldsymbol{v}}_{\tau}\right)^{T}\right)
$$

where, if $\boldsymbol{w}$ is a vector in the tangent space to the surface $\mathcal{S}, \boldsymbol{w}=w^{\alpha} \boldsymbol{\tau}_{\alpha}$, then

$$
\nabla_{\mathcal{S}} \boldsymbol{w} \equiv w_{; \beta}^{\alpha} \boldsymbol{\tau}_{\alpha} \otimes \boldsymbol{\tau}^{\beta}
$$

with $\left\{\boldsymbol{\tau}^{1}, \boldsymbol{\tau}^{2}\right\}$ the reciprocal basis of $\left\{\boldsymbol{\tau}_{1}, \boldsymbol{\tau}_{2}\right\}$, and

$$
w_{; \beta}^{\alpha} \equiv \frac{\partial w^{\alpha}}{\partial \xi^{\beta}}+\Gamma_{\gamma \beta}^{\alpha} w^{\gamma}, \quad \Gamma_{\gamma \beta}^{\alpha} \equiv \phi^{\alpha \sigma} \frac{\partial^{2} x_{i}}{\partial \xi^{\beta} \partial \xi^{\gamma}} \frac{\partial x_{i}}{\partial \xi^{\sigma}}, \quad \phi^{\alpha \sigma} \equiv \boldsymbol{\tau}^{\alpha} \cdot \boldsymbol{\tau}^{\sigma} .
$$

The surface gradient $\nabla_{\mathcal{S}} \boldsymbol{w}$ is an endomorphism of the tangent space to $\mathcal{S}$ at $\boldsymbol{\xi}$.

So, let $\lambda_{1}, \lambda_{2}$ be the eigenvalues of the mean surface stretching, and let $\boldsymbol{f}_{1}, \boldsymbol{f}_{2}$ be corresponding (for the moment arbitrary) orthonormal eigenvectors ${ }^{3}$. We shall propose three models for $\overline{\boldsymbol{\sigma}}_{s}^{\star}$. Common to these models is the assumption that, if $\lambda_{1} \neq \lambda_{2}$ (in which case, there are two principal directions of $\boldsymbol{D}_{\mathcal{S}} \overline{\boldsymbol{v}}_{\tau}$ uniquely defined), two principal directions of $\overline{\boldsymbol{\sigma}}_{s}^{\star}$, parallel to the topography, coincide with the principal directions of the mean surface stretching. Three different assumptions on the corresponding eigenvalues will differentiate three models of solid constituents. The case $\lambda_{1}=\lambda_{2}$ will be discussed separately for each model.

Before proceeding to state the models, we show how $\overline{\mathbf{P}}_{s}$ can be deduced from our assumptions. Thus, we denote by $\mathbf{C} \equiv\left(C_{\beta}^{\alpha}\right)$ the change of basis matrix from $\left\{\boldsymbol{\tau}_{1}, \boldsymbol{\tau}_{2}\right\}$ to the basis $\left\{\boldsymbol{f}_{1}, \boldsymbol{f}_{2}\right\}$ of the tangent space to $\mathcal{S}$, i.e.,

$$
\boldsymbol{f}_{\beta}=C_{\beta}^{\alpha} \boldsymbol{\tau}_{\alpha}, \quad \beta \in\{1,2\}
$$

and mention that

$$
\mathbf{C C}^{T}=\mathbf{M}_{0}
$$

which holds since the basis $\left\{\boldsymbol{f}_{1}, \boldsymbol{f}_{2}\right\}$ is orthonormal and $\boldsymbol{\tau}_{\alpha} \cdot \boldsymbol{\tau}_{\beta}$ are the entries of the matrix $\mathbf{M}_{0}^{-1}$. Now, by representing $\overline{\boldsymbol{\sigma}}_{s}^{\star}$ in terms of $\left\{\boldsymbol{f}_{1}, \boldsymbol{f}_{2}, \boldsymbol{n}\right\}$ as

$$
\overline{\boldsymbol{\sigma}}_{s}^{\star}=\tilde{\sigma}_{s}^{\alpha \beta} \boldsymbol{f}_{\alpha} \otimes \boldsymbol{f}_{\beta}-\bar{p}_{s} \boldsymbol{n} \otimes \boldsymbol{n},
$$

see definition $(8.8)_{2}$ of $\overline{\boldsymbol{\sigma}}_{s}^{\star}$, we have the relation

$$
\left(\bar{\sigma}^{\star \alpha \beta}\right)=\mathbf{C}\left(\tilde{\sigma}^{\alpha \beta}\right) \mathbf{C}^{T}
$$

which combined with $(8.8)_{3}$ gives

$$
\overline{\mathbf{P}}_{s}=\bar{p}_{s} \mathbf{M}_{0}+\mathbf{C}\left(\tilde{\sigma}^{\alpha \beta}\right) \mathbf{C}^{T} .
$$

Our assumptions on $\overline{\boldsymbol{\sigma}}_{s}^{\star}$ will immediately give $\left(\tilde{\sigma}^{\alpha \beta}\right)$, and hence $\overline{\mathbf{P}}_{s}$ can be deduced from relation (8.10). Finally, note that (8.10) holds, in fact, for any orthonormal vectors $\boldsymbol{f}_{1}, \boldsymbol{f}_{2}$ in the tangent space to $\mathcal{S}$, and not necessarily for eigenvectors of $\boldsymbol{D}_{\mathcal{S}} \overline{\boldsymbol{v}}_{\tau}$.

\footnotetext{
${ }^{3}$ The matrix $\left(\mathcal{D}_{\beta}^{\alpha}\right)$ in the representation $\boldsymbol{D}_{\mathcal{S}} \overline{\boldsymbol{v}}_{\tau}=\mathcal{D}_{\beta}^{\alpha} \boldsymbol{\tau}_{\alpha} \otimes \boldsymbol{\tau}^{\beta}$ is needed to obtain the eigenvalues and eigenvectors of $\boldsymbol{D}_{\mathcal{S}} \overline{\boldsymbol{v}}_{\tau}$, and it has been deduced in Luca et al. [11].
} 


\section{Model 1 of the solid constituent}

If $\lambda_{1} \neq \lambda_{2}$, we suppose that the eigenvectors $\boldsymbol{f}_{1}, \boldsymbol{f}_{2}$ of $\boldsymbol{D}_{\delta} \overline{\boldsymbol{v}}_{\tau}$ are eigenvectors of $\overline{\boldsymbol{\sigma}}_{s}^{\star}$ corresponding to the eigenvalue $-\bar{p}_{s}$, that is,

$$
\overline{\boldsymbol{\sigma}}_{s}^{\star}=-\bar{p}_{s} \boldsymbol{f}_{\alpha} \otimes \boldsymbol{f}_{\alpha}-\bar{p}_{s} \boldsymbol{n} \otimes \boldsymbol{n}=-\bar{p}_{s} \mathbf{1} .
$$

We also assume that $\overline{\boldsymbol{\sigma}}_{s}^{\star}=-\bar{p}_{s} \mathbf{1}$ holds if $\lambda_{1}=\lambda_{2}$, and thus, up to terms of order $O\left(\epsilon^{1+\gamma}\right)$, the granular material is modeled as an inviscid fluid. The stresses $\tilde{\sigma}^{\alpha \beta}$ are then given by the diagonal matrix $\left(\tilde{\sigma}^{\alpha \beta}\right)=-\bar{p}_{s} \mathbf{I}$, and from (8.10) and (8.9) we deduce that $\overline{\mathbf{P}}_{s}=\mathbf{0}$. Therefore, the tangential momentum balance equation (7.10) corresponding to the solid constituent emerges as

$$
\begin{array}{rl}
\frac{\partial}{\partial t}\{ & \left.J_{0} h\left(n_{1}^{s}-m_{1}^{s} \Omega h\right) \bar{\nu} \mathbf{F} \overline{\mathbf{v}}\right\}+\operatorname{Div}\left\{J_{0} h \mathbf{F}\left[\left(n_{2}^{s}-m_{3}^{s} \Omega h\right) \bar{\nu} \overline{\mathbf{v}} \otimes \overline{\mathbf{v}}+\bar{p}_{s} \mathbf{M}_{0}\right]\right\} \\
=-J_{0} & h(\tan \delta)\left\{c+m_{2}^{s} a_{s}\right\}_{+} \bar{\nu} \mathbf{F} \operatorname{sgn} \overline{\mathbf{v}} \\
& -J_{0} h\left\{\left(c+n_{2}^{s} a_{s}-\frac{1}{2} m_{3}^{s} h \tilde{a}_{s}\right) \bar{\nu} \mathbf{I}+\bar{p}_{s} \mathbf{F} \mathbf{W} \mathbf{F}^{-1}\right\} \mathbf{s} \\
& -\frac{1}{2} J_{0} h^{2} m_{3}^{s} \bar{\nu} \frac{\partial}{\partial \xi^{\alpha}}\left(\mathbf{F} \mathbf{W} \mathbf{F}^{-1}\right) \mathbf{F}(\overline{\mathbf{v}} \otimes \overline{\mathbf{v}}) \mathbf{e}_{\alpha}+J_{0} h^{2} \beta_{s} \bar{\nu} \mathbf{F} \mathbf{W} \overline{\mathbf{v}} \\
& +\frac{J_{0} h^{2}}{2\left(c_{0} \bar{\nu}+1-\bar{\nu}\right)}\left\{c+m_{3}^{s}(1-\bar{\nu}) a_{s}+m_{3}^{f} \bar{\nu} a_{f}\right\} \mathbf{F M}_{0} \operatorname{Grad} \bar{\nu} \\
& +c_{D} J_{0} h\left\{\left(1-m_{1}^{f} \Omega h\right) \mathbf{F} \overline{\mathbf{u}}-\left(1-m_{1}^{s} \Omega h\right) \mathbf{F} \overline{\mathbf{v}}\right\}+O\left(\epsilon^{2+\gamma}\right) .
\end{array}
$$

For a bed surface with curvature $\mathcal{H}=O\left(\epsilon^{\gamma}\right)$, equation (8.12) reduces to

$$
\begin{aligned}
& \frac{\partial}{\partial t}\left\{J_{0} h n_{1}^{s} \bar{\nu} \mathbf{F} \overline{\mathbf{v}}\right\}+\operatorname{Div}\left\{J_{0} h \mathbf{F}\left[n_{2}^{s} \bar{\nu} \overline{\mathbf{v}} \otimes \overline{\mathbf{v}}+\bar{p}_{s} \mathbf{M}_{0}\right]\right\} \\
&=-J_{0} h(\tan \delta)\left\{c+m_{2}^{s} a_{s}\right\}_{+} \bar{\nu} \mathbf{F} \operatorname{sgn} \overline{\mathbf{v}}-J_{0} h\left(c+n_{2}^{s} a_{s}\right) \bar{\nu} \mathbf{s} \\
& \quad+\frac{c J_{0} h^{2}}{2\left(c_{0} \bar{\nu}+1-\bar{\nu}\right)} \mathbf{F M}_{0} \operatorname{Grad} \bar{\nu}+c_{D} J_{0} h(\mathbf{F} \overline{\mathbf{u}}-\mathbf{F} \overline{\mathbf{v}})+O\left(\epsilon^{2+\gamma}\right),
\end{aligned}
$$

where $\bar{p}_{s}$ is given by $(7.12)_{1}$. If, moreover, $\mathcal{H}=O(\epsilon)$, the term $m_{2}^{s} a_{s}$ in the expression of the basal shear stress can also be neglected. However, the influence of the curvature of the basal surface in (8.13) is still present, via $a_{s}$ entering the coefficient of $\mathbf{s}$. In both equations (8.4) and (8.12), the extra stresses are present only as basal shear stresses, modeled by a viscous friction law for the fluid, and by a Coulomb friction law for the solid. That is, the rheology of the mixture components is so weak that it manifests itself in the governing shallow avalanche equations only through the coefficients of Boussinesq type, the sliding parameter $\chi_{f}$, the viscous friction coefficient $\tilde{c}$, the basal friction angle $\delta$, and the permeability $c_{D}$. All these phenomenological parameters are also present in the next two models.

\section{Model 2 of the solid constituent}

Now, again with $\lambda_{1} \neq \lambda_{2}$, we suppose that $\boldsymbol{f}_{1}, \boldsymbol{f}_{2}$ are eigenvectors of $\overline{\boldsymbol{\sigma}}_{s}^{\star}$ corresponding to the eigenvalue $-k \bar{p}_{s}$, that is,

$$
\overline{\boldsymbol{\sigma}}_{s}^{\star}=-k \bar{p}_{s} \boldsymbol{f}_{\alpha} \otimes \boldsymbol{f}_{\alpha}-\bar{p}_{s} \boldsymbol{n} \otimes \boldsymbol{n},
$$

where the earth pressure coefficient $k$ is defined as

$$
k \equiv\left\{\begin{array}{lll}
k_{\text {act }}^{1}, & \text { if } & \lambda_{1}+\lambda_{2} \geq 0 \\
k_{\text {pass }}^{1}, & \text { if } & \lambda_{1}+\lambda_{2}<0
\end{array}\right.
$$


with

$$
k_{\text {act } / \text { pass }}^{1} \equiv \frac{2}{\cos ^{2} \varphi}\left\{1 \mp \sqrt{1-\sec ^{2} \delta \cos ^{2} \varphi}\right\}-1 .
$$

In the above, the constant $\varphi$ is the internal angle of friction, $\varphi>\delta$, and the minus sign corresponds to $k_{\text {act }}^{1}$, while the plus refers to $k_{\text {pass }}^{1}$. Moreover, recalling that $\bar{p}_{s}=O(\epsilon)$ and that the idea of introducing $\overline{\boldsymbol{\sigma}}_{s}^{\star}$ in (8.8) was to collect the terms $O(\epsilon)$ of $\overline{\boldsymbol{\sigma}}_{s}$, we can approximate the earth pressure coefficients $k_{\text {act/pass }}^{1}$ arising in (8.14) as follows. With $\tan \delta=O\left(\epsilon^{\gamma}\right)$ and values of the internal angle of friction $\varphi \in\left(30^{\circ}, 40^{\circ}\right)$, see, e.g., Pudasaini and Hutter [22], we have

$$
\sqrt{1-\sec ^{2} \delta \cos ^{2} \varphi}=\sin \varphi+O\left(\epsilon^{2 \gamma}\right)
$$

and hence from (8.15) we deduce

$$
k_{\text {act } / \text { pass }}^{1}=\tilde{k}_{\text {act } / \text { pass }}^{1}+O\left(\epsilon^{2 \gamma}\right), \quad \tilde{k}_{\text {act } / \text { pass }}^{1} \equiv 2 \sec ^{2} \varphi-1 \mp 2 \sec \varphi \tan \varphi .
$$

Therefore, apart from the negligible terms, (8.14) emerges as

$$
\overline{\boldsymbol{\sigma}}_{s}^{\star}=-\tilde{k} \bar{p}_{s} \boldsymbol{f}_{\alpha} \otimes \boldsymbol{f}_{\alpha}-\bar{p}_{s} \boldsymbol{n} \otimes \boldsymbol{n},
$$

where

$$
\tilde{k} \equiv\left\{\begin{array}{lll}
\tilde{k}_{a c t}^{1}, & \text { if } & \lambda_{1}+\lambda_{2} \geq 0 \\
\tilde{k}_{\text {pass }}^{1}, & \text { if } & \lambda_{1}+\lambda_{2}<0 .
\end{array}\right.
$$

Now, since $\boldsymbol{f}_{\alpha} \otimes \boldsymbol{f}_{\alpha}=\mathbf{1}-\boldsymbol{n} \otimes \boldsymbol{n}$, it is clear that (8.17) makes also sense for the case $\lambda_{1}=\lambda_{2}$, and that in (8.17) we can take any two orthonormal vectors $\boldsymbol{f}_{1}, \boldsymbol{f}_{2}$ in the tangent space to $\mathcal{S}$. Thus, for Model 2 of the solid constituent we take (8.17), in which $\boldsymbol{f}_{1}, \boldsymbol{f}_{2}$ are arbitrary orthonormal vectors tangent to $\mathcal{S}^{4}$.

Since (8.17) implies $\left(\tilde{\sigma}^{\alpha \beta}\right)=-\tilde{k}_{\bar{p}} \mathbf{I}$, relations (8.10) and (8.9) yield

$$
\overline{\mathbf{P}}_{s}=(1-\tilde{k}) \bar{p}_{s} \mathbf{M}_{0} \text {. }
$$

This expression of $\overline{\mathbf{P}}_{s}$ is inserted into the tangential momentum balance equation (7.10). If we also recall that the term containing $\overline{\mathbf{p}}_{s}$ is negligible, and that the basal shear stress is given by (8.7), we have

$$
\begin{array}{rl}
\frac{\partial}{\partial t}\{ & \left.J_{0} h\left(n_{1}^{s}-m_{1}^{s} \Omega h\right) \bar{\nu} \mathbf{F} \overline{\mathbf{v}}\right\} \\
& +\operatorname{Div}\left\{J_{0} h \mathbf{F}\left[\left(n_{2}^{s}-m_{3}^{s} \Omega h\right) \bar{\nu} \overline{\mathbf{v}} \otimes \overline{\mathbf{v}}+\tilde{k} \bar{p}_{s} \mathbf{M}_{0}\right]\right\} \\
=-J_{0} & h(\tan \delta)\left\{c+m_{2}^{s} a_{s}\right\}_{+} \bar{\nu} \mathbf{F} \operatorname{sgn} \overline{\mathbf{v}}+2(1-\tilde{k}) J_{0} \Omega h \bar{p}_{s} \mathbf{s} \\
& -J_{0} h\left\{\left(c+n_{2}^{s} a_{s}-\frac{1}{2} m_{3}^{s} h \tilde{a}_{s}\right) \bar{\nu} \mathbf{I}+\bar{p}_{s} \mathbf{F} \mathbf{W} \mathbf{F}^{-1}\right\} \mathbf{s} \\
& -\frac{1}{2} J_{0} h^{2} m_{3}^{s} \bar{\nu} \frac{\partial}{\partial \xi^{\alpha}}\left(\mathbf{F W} \mathbf{F}^{-1}\right) \mathbf{F}(\overline{\mathbf{v}} \otimes \overline{\mathbf{v}}) \mathbf{e}_{\alpha}+J_{0} h^{2} \beta_{s} \bar{\nu} \mathbf{F W} \overline{\mathbf{v}} \\
& +\frac{J_{0} h^{2}}{2\left(c_{0} \bar{\nu}+1-\bar{\nu}\right)}\left\{c+m_{3}^{s}(1-\bar{\nu}) a_{s}+m_{3}^{f} \bar{\nu} a_{f}\right\} \mathbf{F} \mathbf{M}_{0} \operatorname{Grad} \bar{\nu} \\
& +c_{D} J_{0} h\left\{\left(1-m_{1}^{f} \Omega h\right) \mathbf{F} \overline{\mathbf{u}}-\left(1-m_{1}^{s} \Omega h\right) \mathbf{F} \overline{\mathbf{v}}\right\}+O\left(\epsilon^{2+\gamma}\right) .
\end{array}
$$

\footnotetext{
${ }^{4}$ We could have simply postulated (8.11) and (8.14) (in which $\boldsymbol{f}_{1}, \boldsymbol{f}_{2}$ are any two orthonormal vectors tangent to $\mathcal{S}$ ), which avoids the discussion of the cases where $\lambda_{1} \neq \lambda_{2}$ and $\lambda_{1}=\lambda_{2}$. However, the reasoning followed in the paper points out the common features of the models $(8.11),(8.17)$ and the forthcoming (8.21).
} 
From (8.19) one can easily deduce the tangential momentum balance equation corresponding to a bottom surface with small curvature, see also (8.13). Model 2 is an adaption to the case of an arbitrary bed surface of the model due to Iverson and Denlinger [9].

\section{Model 3 of the solid constituent}

Finally, we propose yet another closure relation for $\overline{\boldsymbol{\sigma}}_{s}^{\star}$. Assuming $\lambda_{1} \neq \lambda_{2}$, we require the ordering of the vectors of the basis $\left\{\boldsymbol{f}_{1}, \boldsymbol{f}_{2}\right\}$ to be so as to have $\left|w^{1}\right| \geq\left|w^{2}\right|$, where $w^{1}, w^{2}$ are the components of $\overline{\boldsymbol{v}}_{\tau}$ with respect to this basis, i.e., $\overline{\boldsymbol{v}}_{\tau}=w^{\alpha} \boldsymbol{f}_{\alpha}$. Moreover, we suppose that $\boldsymbol{f}_{1}, \boldsymbol{f}_{2}$ are eigenvectors of $\overline{\boldsymbol{\sigma}}_{s}^{\star}$ corresponding to the eigenvalues $-k_{1} \bar{p}_{s}$ and $-k_{2} \bar{p}_{s}$, respectively. That is,

$$
\overline{\boldsymbol{\sigma}}_{s}^{\star}=-k_{1} \bar{p}_{s} \boldsymbol{f}_{1} \otimes \boldsymbol{f}_{1}-k_{2} \bar{p}_{s} \boldsymbol{f}_{2} \otimes \boldsymbol{f}_{2}-\bar{p}_{s} \boldsymbol{n} \otimes \boldsymbol{n} .
$$

Here the earth pressure coefficients $k_{1}, k_{2}$ are given by

$$
k_{1}=\left\{\begin{array}{ll}
k_{\text {act }}^{1}, & \text { if } \quad \lambda_{1} \geq 0 \\
k_{\text {pass }}^{1}, & \text { if } \quad \lambda_{1}<0,
\end{array} \quad k_{2}= \begin{cases}k_{\text {act }}^{2}, & \text { if } \quad \lambda_{2} \geq 0 \\
k_{\text {pass }}^{2}, & \text { if } \quad \lambda_{2}<0\end{cases}\right.
$$

with $k_{\text {act/pass }}^{1}$ defined in (8.15) and

$$
k_{a c t / p a s s}^{2} \equiv \frac{1}{2}\left\{k_{a c t / p a s s}^{1}+1 \mp \sqrt{\left(k_{\text {act } / p a s s}^{1}-1\right)^{2}+4 \tan ^{2} \delta}\right\},
$$

with the same sign rule as in (8.15) and the choice of $k_{a c t}^{1}$ and $k_{\text {pass }}^{1}$ as depending on whether $\lambda_{1} \geq 0$ or $\lambda_{1}<0$, respectively. Like for Model 2 , the earth pressure coefficients $k_{1}, k_{2}$ can be approximated as

$$
k_{1}=\tilde{k}_{1}+O\left(\epsilon^{2 \gamma}\right), \quad k_{2}=\tilde{k}_{2}+O\left(\epsilon^{2 \gamma}\right),
$$

where

$$
\tilde{k}_{1} \equiv\left\{\begin{array} { l } 
{ \tilde { k } _ { \text { act } } ^ { 1 } , \quad \text { if } \quad \lambda _ { 1 } \geq 0 } \\
{ \tilde { k } _ { \text { pass } } ^ { 1 } , \quad \text { if } \quad \lambda _ { 1 } < 0 , }
\end{array} \quad \tilde { k } _ { 2 } \equiv \left\{\begin{array}{l}
\tilde{k}_{\text {act }}^{1}, \quad \text { if } \quad \lambda_{1} \geq 0, \lambda_{2} \geq 0 \\
\tilde{k}_{\text {pass }}^{1}, \quad \text { if } \quad \lambda_{1}<0, \lambda_{2}<0 \\
1, \quad \text { if } \quad \lambda_{1} \geq 0, \lambda_{2}<0 \text { or } \quad \lambda_{1}<0, \quad \lambda_{2} \geq 0
\end{array}\right.\right.
$$

and hence, apart from the negligible terms, assumption (8.20), stated for the case $\lambda_{1} \neq \lambda_{2}$, emerges as

$$
\overline{\boldsymbol{\sigma}}_{s}^{\star}=-\tilde{k}_{1} \bar{p}_{s} \boldsymbol{f}_{1} \otimes \boldsymbol{f}_{1}-\tilde{k}_{2} \bar{p}_{s} \boldsymbol{f}_{2} \otimes \boldsymbol{f}_{2}-\bar{p}_{s} \boldsymbol{n} \otimes \boldsymbol{n},
$$

or, explicitly,

$$
\overline{\boldsymbol{\sigma}}_{s}^{\star}=\left\{\begin{array}{l}
-\tilde{k}_{a c t}^{1} \bar{p}_{s} \boldsymbol{f}_{\alpha} \otimes \boldsymbol{f}_{\alpha}-\bar{p}_{s} \boldsymbol{n} \otimes \boldsymbol{n}, \quad \text { if } \quad \lambda_{1} \geq 0, \quad \lambda_{2} \geq 0 \\
-\tilde{k}_{\text {pass }}^{1} \bar{p}_{s} \boldsymbol{f}_{\alpha} \otimes \boldsymbol{f}_{\alpha}-\bar{p}_{s} \boldsymbol{n} \otimes \boldsymbol{n}, \quad \text { if } \quad \lambda_{1}<0, \quad \lambda_{2}<0 \\
-\tilde{k}_{a c t}^{1} \bar{p}_{s} \boldsymbol{f}_{1} \otimes \boldsymbol{f}_{1}-\bar{p}_{s} \boldsymbol{f}_{2} \otimes \boldsymbol{f}_{2}-\bar{p}_{s} \boldsymbol{n} \otimes \boldsymbol{n}, \quad \text { if } \quad \lambda_{1} \geq 0, \quad \lambda_{2}<0 \\
-\tilde{k}_{\text {pass }}^{1} \bar{p}_{s} \boldsymbol{f}_{1} \otimes \boldsymbol{f}_{1}-\bar{p}_{s} \boldsymbol{f}_{2} \otimes \boldsymbol{f}_{2}-\bar{p}_{s} \boldsymbol{n} \otimes \boldsymbol{n}, \quad \text { if } \quad \lambda_{1}<0, \quad \lambda_{2} \geq 0 .
\end{array}\right.
$$


Note, in passing, that $\tilde{k}_{\text {act }}^{1}<1$ and $\tilde{k}_{\text {pass }}^{1}>1$. Now, it is important to realize that $(8.21)_{1,2}$ make sense for the case $\lambda_{1}=\lambda_{2}$, and that the orthonormal vectors $\boldsymbol{f}_{1}, \boldsymbol{f}_{2}$ therein can be chosen arbitrarily in the tangent space to $\mathcal{S}$. Thus, for Model 3 of the solid constituent we take (8.21), where $\boldsymbol{f}_{1}, \boldsymbol{f}_{2}$ arising in $(8.21)_{1,2}$ are orthonormal vectors tangent to $\mathcal{S}$ (be they eigenvectors of $\boldsymbol{D}_{\mathcal{S}} \overline{\boldsymbol{v}}_{\tau}$ or not), and $\boldsymbol{f}_{1}, \boldsymbol{f}_{2}$ in $(8.21)_{3,4}$ are eigenvectors of $\boldsymbol{D}_{\delta} \overline{\boldsymbol{v}}_{\tau}$, ordered as indicated above (clearly, in $(8.21)_{3,4}$ we have $\left.\lambda_{1} \neq \lambda_{2}\right)$.

Since (8.21) implies that

$$
\left(\tilde{\sigma}^{\alpha \beta}\right)=-\bar{p}\left(\begin{array}{cc}
\tilde{k}_{1} & 0 \\
0 & \tilde{k}_{2}
\end{array}\right)
$$

from (8.10) we deduce that

$$
\overline{\mathbf{P}}_{s}=\bar{p}_{s} \mathbf{M}_{0}-\bar{p}_{s} \mathbf{C}\left(\begin{array}{cc}
\tilde{k}_{1} & 0 \\
0 & \tilde{k}_{2}
\end{array}\right) \mathbf{C}^{T}
$$

which has to be inserted into the tangential momentum balance equation (7.10). This last model, essentially proposed in Luca et al. [11], is a version adapted to arbitrary topography of the Savage-Hutter model [24], and hence of Mohr-Coulomb type. Clearly, the bulk stresses in Models 2 and 3 of solid constituent depend on the internal angle of friction, but are independent of the basal friction angle.

We summarize the model equations of the binary mixture in the following

Proposition 8.1. Suppose that the avalanche mass is modeled as a solid-fluid mixture for which mass productions are not present. Moreover, let

(i) the interaction force be given by (7.3) with the drag coefficient $c_{D}=O(1)$,

(ii) the pore fluid be modeled by the Newtonian/non-Newtonian law (8.1) with viscosity $\eta=O\left(\epsilon^{2+\gamma}\right)$, and by the bottom viscous friction shown in (8.2) with $\tilde{c}=O\left(\epsilon^{1+\gamma}\right)$,

(iii) the solid constituent be characterized by the bottom Coulomb friction given in (8.5) with $\tan \delta=O\left(\epsilon^{\gamma}\right)$, and for which the stresses in the bulk material are described by one of the models 1 to 3.

Then, the governing equations describing the motion of the avalanche mass on arbitrary topography are as follows:

1) Model 1 of binary mixture: (7.5), (7.6), (7.8), (8.12), (8.4);

2) Model 2 of binary mixture: (7.5), (7.6), (7.8), (8.19), (8.4);

3) Model 3 of binary mixture: (7.5), (7.6), (7.8), (7.10) (in which $\overline{\mathbf{p}}_{\text {s }}$ is negligibly small and the stresses (8.7), (8.22) are accounted for), (8.4).

Before ending this section we mention that the approach here did not make necessary the introduction of the saturation pressure, which often enters a mixture theory due to the saturation constraint (3.3). The reason for this is that the interaction force $\stackrel{+}{m}$ given by (7.1) shows no explicit dependence on the saturation pressure, as, e.g., in Passman, Nunziato and Walsh [16]. The closure relations for both stress tensors $\boldsymbol{\sigma}_{s, f}$ neither do depend explicitly on the saturation pressure. The same situation arises in Pitman and Le [18]. This is, however, different for some models in Schneider and Hutter [25]. 


\section{Limiting equilibrium}

Now we are interested in the analysis of the conditions satisfied by $\bar{\nu}, h$ when the avalanche mass described in Proposition 8.1 is in equilibrium, that is, when

$$
\frac{\partial \bar{\nu}}{\partial t}=0, \quad \overline{\mathbf{v}}=\mathbf{0}, \quad \overline{\mathbf{u}}=\mathbf{0}, \quad \frac{\partial h}{\partial t}=0
$$

hold at any $\boldsymbol{\xi}$, and for any $t$ in some interval. However, we must draw attention to the fact that, when satisfying (9.1), the solutions $\bar{\nu}, h$ of the model equations may have no physical reality. Indeed, usually a phase separation process initiates when the solid-fluid mixture reaches the rest state, or, in the approach developed here, it is assumed that each point of the avalanche body is occupied simultaneously by both constituent particles. Besides, the closure relations (8.17) and (8.21) are intended to describe the stress state in a rapidly flowing avalanche mass, and hence other closure relations may be necessary to describe the constitutive behaviour of the solidfluid mixture at rest. We merely expect the solutions $\bar{\nu}, h$, when satisfying (9.1), to approximate the real volume fraction and avalanche height for a very short time period after the moment when the avalanche stops to flow; in this sense we refer to (9.1) as describing a limiting equilibrium, see also the comments in Iverson and Denlinger [9]. But it remains for experiments and numerics to validate this conjecture. Here we only derive the equations satisfied by $\bar{\nu}, h$, under assumptions (9.1) and corresponding to Models 1 to 3 .

With respect to Models 2 and 3 we make the following remark. The earth pressure coefficient $\tilde{k}$ is discontinuous at $\lambda_{1}+\lambda_{2}=0$, and $\tilde{k}_{1}, \tilde{k}_{2}$ have also discontinuity points. Moreover, the values of these coefficients at the discontinuity points were, in general, arbitrarily assigned (e.g., we could have taken $k=k_{\text {pass }}^{1}$ for $\lambda_{1}+\lambda_{2}=0$ ), since the values of these coefficients were deduced for $\lambda_{1} \neq 0, \lambda_{2} \neq 0$, by combining the MohrCoulomb theory with the Rankine model of soil mechanics, see Hutter et al. [7]. In order to reduce the computational difficulties connected to these discontinuities, it is therefore not a bad idea to use regularizing functions $\hat{k}, \hat{k}_{1}, \hat{k}_{2}$ instead of $\tilde{k}, \tilde{k}_{1}, \tilde{k}_{2}$, respectively, as Tai and Gray [26], and Tai, Hutter and Gray [27] have done for the earth pressure coefficients $k_{1}, k_{2}$. In doing so, the arbitrariness of the earth pressure coefficients at the discontinuity points is replaced by the arbitrariness of the choice of the regularizing functions. However, the values of $\hat{k}, \hat{k}_{1}, \hat{k}_{2}$ at $\lambda_{1}=\lambda_{2}=0$ play an important role in the description of the limiting equilibrium, and hence they must be carefully selected. Indeed, at equilibrium $\boldsymbol{D}_{\delta} \overline{\boldsymbol{v}}_{\tau}=\boldsymbol{O}$, and hence the stress tensor corresponding to Models 2 and 3 has the form

$$
\overline{\boldsymbol{\sigma}}_{s}^{\star}=-k_{0} \bar{p}_{s} \boldsymbol{f}_{\alpha} \otimes \boldsymbol{f}_{\alpha}-\bar{p}_{s} \boldsymbol{n} \otimes \boldsymbol{n},
$$

where $k_{0}$ is the value of $\hat{k}$ or $\hat{k}_{1}$, respectively, at $\lambda_{1}=\lambda_{2}=0$, and $\boldsymbol{f}_{1}, \boldsymbol{f}_{2}$ are orthonormal vectors tangent to $\mathcal{S}$. It is then clear that the study of the limiting equilibrium can give information on $k_{0}$. We note that Iverson and Denlinger [9] assumed $k_{0}=1$ in their model.

Thus, we understand that in Models 2 and 3 the earth pressure coefficients are regularized and exploit the depth-integrated mass and tangential momentum balance equations corresponding to the solid and fluid constituents under assumptions (9.1), accounting for (9.2). Taking $k_{0}=1$ in (9.2) we obtain the stress tensor corresponding to Model 1 , and so the next calculations, conducted for arbitrary $k_{0}$, envisage all three models. 
To begin with, we note that under assumptions $(9.1)$ the mean pressures $\bar{p}_{s}, \bar{p}_{f}$ are given by

$$
\bar{p}_{s}=\frac{1}{2} h c \bar{\nu}+O\left(\epsilon^{1+\gamma}\right), \quad \bar{p}_{f}=\frac{1}{2} h c(1-\bar{\nu})+O\left(\epsilon^{1+\gamma}\right),
$$

see (7.5), and that the depth-averaged mass balance equations (7.6), (7.8) are identically satisfied. Then, having in view (9.1), (9.2), the depth-integrated momentum balance equation corresponding to the solid constituent can be deduced from (8.19), in which $\tilde{k}$ is replaced by $k_{0}$, as

$$
\begin{aligned}
& \operatorname{Div}\left\{k_{0} J_{0} h \bar{p}_{s} \mathbf{F M}_{0}\right\} \\
= & -J_{0} h c(\tan \delta) \bar{\nu} \mathbf{F m}+2\left(1-k_{0}\right) J_{0} \Omega h \bar{p}_{s} \mathbf{s} \\
& -J_{0} h\left\{c \bar{\nu} \mathbf{I}+\bar{p}_{s} \mathbf{F} \mathbf{W F} \mathbf{F}^{-1}\right\} \mathbf{s}+\frac{J_{0} h^{2} c}{2\left(c_{0} \bar{\nu}+1-\bar{\nu}\right)} \mathbf{F M}_{0} \operatorname{Grad} \bar{\nu}+O\left(\epsilon^{2+\gamma}\right),
\end{aligned}
$$

and by using (9.1), the depth-integrated momentum balance equation (8.4) corresponding to the fluid constituent can be written as

$$
\begin{aligned}
& \operatorname{Div}\left\{J_{0} h \bar{p}_{f} \mathbf{F} \mathbf{M}_{0}\right\} \\
= & -J_{0} h\left\{c(1-\bar{\nu}) \mathbf{I}+\bar{p}_{f} \mathbf{F} \mathbf{W F} \mathbf{F}^{-1}\right\} \mathbf{s}-\frac{c_{0} J_{0} h^{2} c}{2\left(c_{0} \bar{\nu}+1-\bar{\nu}\right)} \mathbf{F M}_{0} \operatorname{Grad} \bar{\nu}+O\left(\epsilon^{2+\gamma}\right) .
\end{aligned}
$$

We therefore have to exploit (9.4), (9.5), in which (9.3) is accounted for. Thus, using the formulae

$$
\begin{gathered}
\operatorname{Div} \mathbf{F} \mathbf{M}_{0}=\frac{\partial \mathbf{F}}{\partial \xi^{\alpha}} \mathbf{M}_{0} \mathbf{e}_{\alpha}+\mathbf{F} \operatorname{Div} \mathbf{M}_{0} \\
\operatorname{Div} \mathbf{M}_{0}=-\mathbf{F}^{-1} \frac{\partial \mathbf{F}}{\partial \xi^{\alpha}} \mathbf{M}_{0} \mathbf{e}_{\alpha}-\operatorname{tr}\left(\mathbf{F}^{-1} \frac{\partial \mathbf{F}}{\partial \xi^{\alpha}}\right) \mathbf{M}_{0} \mathbf{e}_{\alpha}-\mathbf{W F} \mathbf{F}^{-1} \mathbf{s}-2 \Omega \mathbf{F}^{-1} \mathbf{s} \\
\operatorname{Grad} J_{0}=J_{0} \operatorname{tr}\left(\mathbf{F}^{-1} \frac{\partial \mathbf{F}}{\partial \xi^{\alpha}}\right) \mathbf{e}_{\alpha}+J_{0} \mathbf{H} \mathbf{F}^{-1} \mathbf{s}, \quad \operatorname{Grad} c=-c \mathbf{H F}^{-1} \mathbf{s}
\end{gathered}
$$

the last three of which have been deduced in Luca, Tai and Kuo [10], after a routine calculation equations $(9.4),(9.5)$ can be cast into the form

$$
\begin{aligned}
& k_{0} \bar{\nu} \mathbf{F M}_{0} \operatorname{Grad} h+h \frac{k_{0}\left(c_{0}-1\right) \bar{\nu}+k_{0}-1}{2\left(c_{0} \bar{\nu}+1-\bar{\nu}\right)} \mathbf{F M}_{0} \operatorname{Grad} \bar{\nu} \\
& +\frac{1}{2}\left(1-k_{0}\right) h \bar{\nu} \mathbf{F} \mathbf{W} \mathbf{F}^{-1} \mathbf{s}+(1-\Omega h) \bar{\nu} \mathbf{s} \\
= & -(\tan \delta) \bar{\nu} \mathbf{F} \mathbf{m}+O\left(\epsilon^{1+\gamma}\right), \\
& \mathbf{F M}_{0} \operatorname{Grad} h+\frac{\left(c_{0}-1\right) h}{2\left(c_{0} \bar{\nu}+1-\bar{\nu}\right)} \mathbf{F M}_{0} \operatorname{Grad} \bar{\nu}+(1-\Omega h) \mathbf{s}=O\left(\epsilon^{1+\gamma}\right) .
\end{aligned}
$$

Moreover, insertion of the expression of $\mathbf{F M}_{0} \mathrm{Grad} h$ as deduced from (9.7) into (9.6) transforms the latter into

$$
\begin{aligned}
& \left(1-k_{0}\right)\left\{\frac{h}{2\left(c_{0} \bar{\nu}+1-\bar{\nu}\right)} \mathbf{F M}_{0} \operatorname{Grad}_{\bar{\nu}}-\frac{1}{2} h \bar{\nu} \mathbf{F} \mathbf{W} \mathbf{F}^{-1} \mathbf{s}-(1-\Omega h) \bar{\nu} \mathbf{s}\right\} \\
= & (\tan \delta) \bar{\nu} \mathbf{F} \mathbf{m}+O\left(\epsilon^{1+\gamma}\right) .
\end{aligned}
$$


We recall that $\mathbf{m}$ in (9.8) is specified by the condition $\mathbf{M}_{0}^{-1} \mathbf{m} \cdot \mathbf{m} \leq 1$, and hence (9.8) must be interpreted as the inequality implied by this condition.

Relations (9.7), (9.8) are those that have to be satisfied by $\bar{\nu}$ and $h$ at the limiting equilibrium. We note that equation (9.7) can be written in the form, see (2.1) and definition (2.2) of $\mathbf{M}_{0}$,

$$
c \operatorname{grad} h+\frac{\left(c_{0}-1\right) h c}{2\left(c_{0} \bar{\nu}+1-\bar{\nu}\right)} \operatorname{grad} \bar{\nu}+(1-\Omega h) \operatorname{grad} b=O\left(\epsilon^{1+\gamma}\right) .
$$

For small curvature, $\mathcal{H}=O\left(\epsilon^{\gamma}\right)$, we have grad $c=O\left(\epsilon^{\gamma}\right)$, and hence (9.9) emerges as

$$
\operatorname{grad}(b+h c)+\frac{\left(c_{0}-1\right) h c}{2\left(c_{0} \bar{\nu}+1-\bar{\nu}\right)} \operatorname{grad} \bar{\nu}=O\left(\epsilon^{1+\gamma}\right) .
$$

We close this analysis with the following remarks concerning (9.7) (or equivalently (9.9)) and (9.8).

1) If $k_{0}=1$, condition (9.8) is satisfied and there remains only equation (9.9) to restrict $\bar{\nu}$ and $h$ at equilibrium.

2) If $\operatorname{Grad} \bar{\nu}=\mathbf{0}$ and $k_{0} \neq 1$, both (9.7), (9.8) are restrictions on $h$ and hence no solution may exist for $h$. Thus we expect uniform distribution of $\bar{\nu}$ at equilibrium only if $k_{0}=1$.

3) For very close values of the true densities of both constituents, in the sense that $c_{0}-1=O\left(\epsilon^{\gamma}\right)$, see (4.6) (which is not generally true, e.g., in debris flows, but may be true, e.g., if two fluids are mixed, in which case Model 1 for the "solid" constituent is most likely appropriate), or for $\operatorname{grad} \bar{\nu}=\mathbf{0},(9.9)$ turns into

$$
c \operatorname{grad} h+(1-\Omega h) \operatorname{grad} b=O\left(\epsilon^{1+\gamma}\right) .
$$

This equation was also deduced in Luca, Tai and Kuo [10] for the single constituent avalanche body modeled as an inviscid or viscous fluid. In particular, for small curvature $\mathcal{H}=O\left(\epsilon^{\gamma}\right)$ it emerges as

$$
b+c h=\text { constant }
$$

that is, the equilibrium free surface is horizontal.

4) For $k_{0} \neq 1$ and negligible basal friction of the solid constituent, that is, $\tan \delta=$ $O\left(\epsilon^{1+\gamma}\right)$, condition (9.8) takes the form

$$
\frac{h}{2\left(c_{0} \bar{\nu}+1-\bar{\nu}\right)} \mathbf{F M}_{0} \operatorname{Grad} \bar{\nu}-\frac{1}{2} h \bar{\nu} \mathbf{F} \mathbf{W F}^{-1} \mathbf{s}-(1-\Omega h) \bar{\nu} \mathbf{s}=O\left(\epsilon^{1+\gamma}\right),
$$

which, noticing that

$$
\begin{gathered}
\mathbf{F W F} \mathbf{F}^{-1} \mathbf{s}=\mathbf{F M}_{0} \mathbf{H F}^{-1} \mathbf{s}=\mathbf{F}^{-T} \mathbf{H} \mathbf{F}^{-1} \mathbf{s}-\left(\mathbf{s} \cdot \mathbf{F}^{-T} \mathbf{H} \mathbf{F}^{-1} \mathbf{s}\right) \mathbf{s} \\
\mathbf{F}^{-T} \mathbf{H F}^{-1}=c \operatorname{grad}(\operatorname{grad} b),
\end{gathered}
$$

can be rewritten as

$$
\frac{h c}{2\left(c_{0} \bar{\nu}+1-\bar{\nu}\right)} \operatorname{grad} \bar{\nu}-\frac{1}{2} h c^{3} \bar{\nu} \operatorname{grad}(\operatorname{grad} b) \operatorname{grad} b-(1-\Omega h) \bar{\nu} \operatorname{grad} b=O\left(\epsilon^{1+\gamma}\right) .
$$


This can be used to transform (9.9) into

$$
c \operatorname{grad} h+\frac{1}{2}\left(c_{0}-1\right) h c^{3} \bar{\nu} \operatorname{grad}(\operatorname{grad} b) \operatorname{grad} b+\left(c_{0} \bar{\nu}+1-\bar{\nu}\right)(1-\Omega h) \operatorname{grad} b=O\left(\epsilon^{1+\gamma}\right) .
$$

Therefore, equations (9.11), (9.12) stand for the determination of $\bar{\nu}$ and $h$ for the case $k_{0} \neq 1$ and negligible basal Coulomb friction. With $\mathcal{H}=O\left(\epsilon^{\gamma}\right)$, they emerge as

$$
\begin{gathered}
h c \operatorname{grad} \bar{\nu}-2\left(c_{0} \bar{\nu}+1-\bar{\nu}\right) \bar{\nu} \operatorname{grad} b=O\left(\epsilon^{1+\gamma}\right), \\
\operatorname{grad} h c+\left(c_{0} \bar{\nu}+1-\bar{\nu}\right) \operatorname{grad} b=O\left(\epsilon^{1+\gamma}\right),
\end{gathered}
$$

which are still too complicated for finding an analytic solution.

The remarks above make obvious the fact that the case $k_{0}=1$ is the simplest case which can be considered when regularizing the earth pressure coefficients, but it is equally interesting to take $k_{0} \neq 1$, e.g., $k_{0}=2 \sec ^{2} \varphi-1$, see (8.16).

\section{Conclusions}

In this paper we presented a quasi-general model and three particular models of binary mixtures for shallow debris flows on arbitrary rigid terrain. In doing so, we followed Truesdell's mixture theory and the approach in Luca et al. [11] dealing with a one-constituent avalanche mass. In particular, we used curvilinear coordinates adapted to the topography as introduced by Bouchut and Westdickenberg [1]. A brief review of these coordinates is given in section 2. In section 3 the intrinsic basic equations and boundary conditions are formulated, however, the material laws are not yet specified, so that the terms "solid" and "fluid", which we use to refer to both constituents, are only formally used. In writing these equations the following assumptions are laid down:

- both constituents simultaneously occupy the same domain, bounded below by the basal surface and above by the free surface;

- no mass exchange occurs between constituents, and neither erosion nor deposition are considered;

- the constituents are density preserving;

- there are no voids in the mixture;

- the motion of each constituent is governed by the mass and momentum balance equations, in which the interaction force between constituents is accounted for;

- both constituents have zero normal velocity at the basal surface;

- the free surface is a material surface for each constituent;

- the stresses acting perpendicular to the free surface and corresponding to both constituents are zero.

In section 4, Proposition 4.1, the intrinsic governing equations are written in nondimensional form and in terms of the previously mentioned curvilinear coordinates. In section 5 the exact depth-integrated mass and tangential momentum balance equations corresponding to each constituent are deduced, see Proposition 5.1, and the depth-averaging procedure, followed in the subsequent sections, is described. Part of this procedure is the formulation of ordering approximations, and this issue is addressed in section 6 . The approximations account for

- the shallow geometry of the avalanche body; 
- significant values of the tangential velocities of both constituents;

- deviations both of the tangential velocity from a "plug-flow" profile and of the solid volume fraction from a linear profile, manifested by coefficients of Boussinesq type;

- dynamic assumptions by which, in particular, the stress vector on the free surface is negligibly small.

These approximations are next used to exploit the local mass balance equations, the normal momentum balance equations, and the depth-integrated mass and tangential momentum balance equations, in which the interaction force is given by an ad-hoc closure relation that we have taken from Schneider and Hutter [25]. Proposition 7.1 collects the emerging equations, that stand for the determination of the depth-averaged solid volume fraction, depth-averaged tangential constituent velocities and free surface height, as soon as closure relations for the mean stress tensor corresponding to each constituent are given. Section 8 is devoted to the formulation of these closure relations. Thus, one constituent is supposed to be a Newtonian/nonNewtonian fluid with small viscosity and experiencing bottom friction. If the viscosity is sufficiently small, only the basal shear stress survives in the tangential momentum balance equation, and this is parameterized by a viscous law with a constant friction coefficient. The other constituent is a solid subjected to basal friction of Coulomb type. Moreover, for its bulk stresses three models are proposed. Common to these models is the assumption that two of the principal directions of the mean stress tensor are aligned with the principal directions of the mean surface stretching. What differentiates the models is the statement on the eigenvalues of the mean stress tensor. The simplest case is envisaged by Model 1, which, apart from negligible terms, reduces to the inviscid fluid. Model 2 is an adaption of the Iverson and Denlinger model [9] to arbitrary topography. The most general model is Model 3, which constitutes an adaption of the Savage-Hutter model [24] to arbitrary bed surfaces, and hence of Mohr-Coulomb type. Proposition 8.1 indicates the governing equations corresponding to these three models of a solid-fluid mixture flowing on an arbitrary topography. Finally, in section 9 the conditions satisfied by the solid volume fraction and avalanche depth at equilibrium are deduced, and particular cases of them are discussed. Numerical simulations using the models proposed here are relegated to further papers.

Appendix. Here we prove Proposition 7.1. In doing so, we closely follow the proof of a similar proposition stated for the case of a single phase, see Appendix A in Luca et al. [11]. Thus, we start with the normal momentum balance equation (4.9) corresponding to the solid constituent, which under the scalings in section 6 and assumption $(6.10)_{2}$ emerges as

$$
\begin{array}{r}
\underbrace{\frac{\partial}{\partial t}(J \nu \mathrm{v})}_{O(\epsilon)}+\underbrace{\operatorname{Div}\left\{J\left(\nu \mathbf{v} \mathbf{v}-\mathbf{p}_{s}\right)\right\}}_{O\left(\epsilon^{\gamma}\right)}+\underbrace{\frac{\partial}{\partial \xi}\left(J\left(\nu \mathbf{v}^{2}-T_{s}^{33}\right)\right)}_{O\left(\epsilon^{\gamma}\right)}+\underbrace{J \frac{\partial p_{s}}{\partial \xi}}_{O(1)}+\underbrace{J \Gamma\left(\mathbf{P}_{s}\right)}_{O(\epsilon)}= \\
\underbrace{-J \nu c}_{O(1)}+\underbrace{J \nu \Gamma(\mathbf{v})}_{O(1)}+\underbrace{J \stackrel{+}{\mathrm{m}}}_{O(1)} .
\end{array}
$$

Accounting for $\mathrm{v}=\mathrm{u}=O(\epsilon), c_{D}=O(1)$ in expression $(7.4)_{2}$ of $\stackrel{+}{\mathrm{m}}$, and neglecting 
the terms that are not of order $O(1)$ in the relation above, we obtain

$$
\frac{\partial p_{s}}{\partial \xi}=-\nu c+\nu \Gamma(\mathbf{v})+\left\{\left(1-c_{s}\right) \tilde{p}_{s}+\frac{c_{s}}{c_{0}} \tilde{p}_{f}\right\} \frac{\partial \nu}{\partial \xi}+O\left(\epsilon^{\gamma}\right) .
$$

Moreover, with $p_{s}=\nu \tilde{p}_{s}, c_{s}$ as given by (7.2), and

$$
\Gamma(\mathbf{v})=-\mathbf{H} \cdot(\mathbf{v} \otimes \mathbf{v})+O(\epsilon)
$$

see $(4.5),(2.4)_{2}$, the above equation can be written as

$$
\frac{\partial \tilde{p}_{s}}{\partial \xi}=-c-\mathbf{H} \cdot(\mathbf{v} \otimes \mathbf{v})+\frac{1}{c_{0} \nu+1-\nu}\left(\tilde{p}_{f}-c_{0} \tilde{p}_{s}\right) \frac{\partial \nu}{\partial \xi}+O\left(\epsilon^{\gamma}\right) .
$$

Similarly, from the normal momentum balance equation (4.12) corresponding to the fluid constituent we deduce that

$$
\frac{\partial \tilde{p}_{f}}{\partial \xi}=-c-\mathbf{H} \cdot(\mathbf{u} \otimes \mathbf{u})+\frac{1}{c_{0} \nu+1-\nu}\left(\tilde{p}_{f}-c_{0} \tilde{p}_{s}\right) \frac{\partial \nu}{\partial \xi}+O\left(\epsilon^{\gamma}\right) .
$$

The system (A.1), (A.2) can be solved to deduce the true pressures $\tilde{p}_{s}, \tilde{p}_{f}$ as follows. We multiply equation (A.1) by $\left(-c_{0}\right)$ and add the emerging equation to (A.2). One obtains

$$
\begin{aligned}
& \frac{\partial}{\partial \xi}\left\{\left(c_{0} \nu+1-\nu\right)\left(\tilde{p}_{f}-c_{0} \tilde{p}_{s}\right)\right\} \\
&=\left(c_{0} \nu+1-\nu\right)\left\{\left(c_{0}-1\right) c+c_{0} \mathbf{H} \cdot(\mathbf{v} \otimes \mathbf{v})-\mathbf{H} \cdot(\mathbf{u} \otimes \mathbf{u})\right\}+O\left(\epsilon^{\gamma}\right) .
\end{aligned}
$$

Integrating the preceding equation from $\xi$ to $h(\boldsymbol{\xi}, t)$, with the aid of the boundary conditions (6.9), which appear as

$$
\tilde{p}_{s}=O\left(\epsilon^{1+\gamma}\right), \quad \tilde{p}_{f}=O\left(\epsilon^{1+\gamma}\right) \quad \text { at } \quad \xi=h(\boldsymbol{\xi}, t),
$$

we deduce that

$$
\begin{aligned}
& \left(c_{0} \nu+1-\nu\right)\left(\tilde{p}_{f}-c_{0} \tilde{p}_{s}\right) \\
= & -\int_{\xi}^{h(\boldsymbol{\xi}, t)}\left(c_{0} \nu+1-\nu\right)\left\{\left(c_{0}-1\right) c+c_{0} \mathbf{H} \cdot(\mathbf{v} \otimes \mathbf{v})-\mathbf{H} \cdot(\mathbf{u} \otimes \mathbf{u})\right\} d \xi^{\prime}+O\left(\epsilon^{1+\gamma}\right) .
\end{aligned}
$$

Finally, with $\nu=\bar{\nu}+O(\epsilon)$, we have

$$
\begin{aligned}
& \tilde{p}_{f}-c_{0} \tilde{p}_{s} \\
= & -\int_{\xi}^{h(\boldsymbol{\xi}, t)}\left\{\left(c_{0}-1\right) c+c_{0} \mathbf{H} \cdot(\mathbf{v} \otimes \mathbf{v})-\mathbf{H} \cdot(\mathbf{u} \otimes \mathbf{u})\right\} d \xi^{\prime}+O\left(\epsilon^{1+\gamma}\right)=O(\epsilon) .
\end{aligned}
$$

The expression above and

$$
\frac{1}{c_{0} \nu+1-\nu}=\frac{1}{c_{0} \bar{\nu}+1-\bar{\nu}}+O(\epsilon)
$$

are next substituted into (A.1), (A.2). Thus, noting that

$$
\int_{\xi}^{h(\boldsymbol{\xi}, t)}\left(\tilde{p}_{f}-c_{0} \tilde{p}_{s}\right) \frac{\partial \nu}{\partial \xi^{\prime}} d \xi^{\prime}=O\left(\epsilon^{1+\gamma}\right)
$$


which is deduced by first integrating by parts and then by using (A.5) ${ }^{5}$, (A.3), (6.2), the integration of (A.1), (A.2) from $\xi$ to $h(\boldsymbol{\xi}, t)$ gives the true pressures as

$$
\begin{aligned}
& \tilde{p}_{s}=\tilde{p}_{s}^{\star}+O\left(\epsilon^{1+\gamma}\right), \quad \tilde{p}_{s}^{\star} \equiv c(h-\xi)+\mathbf{H} \cdot \int_{\xi}^{h(\xi, t)} \mathbf{v} \otimes \mathbf{v} d \xi^{\prime}, \\
& \tilde{p}_{f}=\tilde{p}_{f}^{\star}+O\left(\epsilon^{1+\gamma}\right), \quad \tilde{p}_{f}^{\star} \equiv c(h-\xi)+\mathbf{H} \cdot \int_{\xi}^{h(\xi, t)} \mathbf{u} \otimes \mathbf{u} d \xi^{\prime} .
\end{aligned}
$$

The mean values of the pressures $p_{s}, p_{f}$ are then given as in (7.5) by noting that

$$
p_{s}=\bar{\nu} \tilde{p}_{s}^{\star}+O\left(\epsilon^{1+\gamma}\right), \quad p_{f}=\bar{\nu} \tilde{p}_{f}^{\star}+O\left(\epsilon^{1+\gamma}\right)
$$

and that

$$
\begin{aligned}
& \int_{0}^{h(\boldsymbol{\xi}, t)}\left(\int_{\xi}^{h(\xi, t)} \mathbf{v}\left(\xi^{\prime}\right) \otimes \mathbf{v}\left(\xi^{\prime}\right) d \xi^{\prime}\right) d \xi=\int_{0}^{h(\boldsymbol{\xi}, t)}\left(\int_{0}^{\xi^{\prime}} \mathbf{v}\left(\xi^{\prime}\right) \otimes \mathbf{v}\left(\xi^{\prime}\right) d \xi\right) d \xi^{\prime} \\
= & \int_{0}^{h(\boldsymbol{\xi}, t)} \xi^{\prime} \mathbf{v}\left(\xi^{\prime}\right) \otimes \mathbf{v}\left(\xi^{\prime}\right) d \xi^{\prime}=\frac{1}{2} h^{2} m_{3}^{s} \overline{\mathbf{v}} \otimes \overline{\mathbf{v}}+O\left(\epsilon^{2+\gamma}\right),
\end{aligned}
$$

and a similar relation with $\mathbf{u}$ instead of $\mathbf{v}$, hold.

We now refer to the depth-integrated mass balance equation (5.1), in which the integrands are evaluated up to terms $O\left(\epsilon^{2}\right)$. So, with the aid of (6.6) and (6.2) we obtain

$$
J \nu=J_{0}(\nu-2 \Omega \bar{\nu} \xi)+O\left(\epsilon^{2}\right), \quad J \nu \mathbf{v}=J_{0}(\nu \mathbf{v}-2 \Omega \bar{\nu} \xi \mathbf{v})+O\left(\epsilon^{2}\right),
$$

whence, together with $(6.1)_{1},(6.3)_{1}$, we deduce

$$
\begin{gathered}
\int_{0}^{h(\boldsymbol{\xi}, t)} J \nu d \xi=J_{0} h(1-\Omega h) \bar{\nu}+O\left(\epsilon^{3}\right), \\
\int_{0}^{h(\boldsymbol{\xi}, t)} J \nu \mathbf{v} d \xi=J_{0} h\left(n_{1}^{s}-m_{1}^{s} \Omega h\right) \bar{\nu} \overline{\mathbf{v}}+O\left(\epsilon^{2+\gamma}\right),
\end{gathered}
$$

and hence (7.6) follows.

Now, we pass to the depth-integrated tangent momentum balance equation (5.2) and evaluate the integrands up to order $O\left(\epsilon^{1+\gamma}\right)$. The first integral on the left-hand side of (5.2) has already been determined, see $(\text { A.8 })_{2}$. So, we refer to the integral under the divergence operator and notice that, with (6.6), (6.2), $(6.3)_{2}$ and

$$
p_{s}=p_{s}^{\star}+O\left(\epsilon^{1+\gamma}\right)=O(\epsilon), \quad p_{s}^{\star} \equiv \bar{\nu} \tilde{p}_{s}^{\star}, \quad \mathbf{M}=\mathbf{M}_{0}+O(\epsilon), \quad \mathbf{P}_{s}=O(\epsilon),
$$

we deduce

$$
J\left(\nu \mathbf{v} \otimes \mathbf{v}+p_{s} \mathbf{M}-\mathbf{P}_{s}\right)=J_{0}\left\{\nu \mathbf{v} \otimes \mathbf{v}-2 \Omega \bar{\nu} \xi \mathbf{v} \otimes \mathbf{v}+p_{s}^{\star} \mathbf{M}-\mathbf{P}_{s}\right\}+O\left(\epsilon^{1+\gamma}\right),
$$

which implies

$$
\begin{aligned}
& \int_{0}^{h(\boldsymbol{\xi}, t)} J\left(\nu \mathbf{v} \otimes \mathbf{v}+p_{s} \mathbf{M}-\mathbf{P}_{s}\right) d \xi \\
= & J_{0} h\left\{\left(n_{2}^{s}-m_{3}^{s} \Omega h\right) \bar{\nu} \overline{\mathbf{v}} \otimes \overline{\mathbf{v}}+\bar{p}_{s}^{\star} \mathbf{M}_{0}-\overline{\mathbf{P}}_{s}\right\}+O\left(\epsilon^{2+\gamma}\right) .
\end{aligned}
$$

\footnotetext{
${ }^{5}$ What actually counts after the integration by parts is that $\nu=\bar{\nu}+O(\epsilon)$ and $\tilde{p}_{f}-c_{0} \tilde{p}_{s}=O(\epsilon)$.
} 
The next term in $(5.2)$ is

$$
\left.\left(J \mathbf{p}_{s}\right)\right|_{\xi=0}=\left.J_{0} \mathbf{p}_{s}\right|_{\xi=0} .
$$

Then, the term evaluated at $\xi=h$ is of order $O\left(\epsilon^{2+\gamma}\right)$, see (6.5), and hence it can be neglected.

Now, with $(\mathrm{A} .9)_{1,3},(6.6)$ and definition (2.4) of $\mathbf{B}$, from (4.2) we deduce

$$
J \boldsymbol{\Gamma}\left(-p_{s} \mathbf{M}, \mathbf{0}\right)=J_{0} p_{s}^{\star}\left\{\mathbf{F}^{-1} \frac{\partial \mathbf{F}}{\partial \xi^{\alpha}} \mathbf{M}_{0} \mathbf{e}_{\alpha}+2 \Omega \mathbf{F}^{-1} \mathbf{s}\right\}+O\left(\epsilon^{1+\gamma}\right) .
$$

This, together with

$$
J \boldsymbol{\Gamma}\left(\mathbf{P}_{s}, \mathbf{p}_{s}\right)=J_{0}\left\{-\mathbf{F}^{-1} \frac{\partial \mathbf{F}}{\partial \xi^{\alpha}} \mathbf{P}_{s} \mathbf{e}_{\alpha}+2 \mathbf{W} \mathbf{p}_{s}-\left(\mathbf{H} \cdot \mathbf{P}_{s}\right) \mathbf{F}^{-1} \mathbf{s}\right\}+O\left(\epsilon^{1+\gamma}\right),
$$

which can be derived from $(4.3),(4.5)_{1}$ and (6.4), yields

$$
\begin{aligned}
\int_{0}^{h(\boldsymbol{\xi}, t)} J\left\{\boldsymbol{\Gamma}\left(-p_{s} \mathbf{M}, \mathbf{0}\right)+\boldsymbol{\Gamma}\left(\mathbf{P}_{s}, \mathbf{p}_{s}\right)\right\} d \xi \\
=J_{0} h \bar{p}_{s}^{\star}\left\{\mathbf{F}^{-1} \frac{\partial \mathbf{F}}{\partial \xi^{\alpha}} \mathbf{M}_{0} \mathbf{e}_{\alpha}+2 \Omega \mathbf{F}^{-1} \mathbf{s}\right\} \\
\quad-J_{0} h\left\{\mathbf{F}^{-1} \frac{\partial \mathbf{F}}{\partial \xi^{\alpha}} \overline{\mathbf{P}}_{s} \mathbf{e}_{\alpha}-2 \mathbf{W} \overline{\mathbf{p}}_{s}+\left(\mathbf{H} \cdot \overline{\mathbf{P}}_{s}\right) \mathbf{F}^{-1} \mathbf{s}\right\}+O\left(\epsilon^{2+\gamma}\right) .
\end{aligned}
$$

Next, since

$$
\mathbf{B}^{-1}=(\mathbf{I}-\xi \mathbf{W})^{-1} \mathbf{F}^{-1}=(\mathbf{I}+\xi \mathbf{W}) \mathbf{F}^{-1}+O\left(\epsilon^{2}\right),
$$

we have, see $(6.10),(6.2)$,

$$
J \nu \mathbf{b}=-J_{0} c\{(\nu-2 \Omega \bar{\nu} \xi) \mathbf{I}+\xi \bar{\nu} \mathbf{W}\} \mathbf{F}^{-1} \mathbf{s}+O\left(\epsilon^{2}\right)
$$

and therefore the first term on the right-hand side of (5.2) emerges as

$$
\int_{0}^{h(\boldsymbol{\xi}, t)} J \nu \mathbf{b} d \xi=-J_{0} h c \bar{\nu}\left\{(1-\Omega h) \mathbf{I}+\frac{1}{2} h \mathbf{W}\right\} \mathbf{F}^{-1} \mathbf{s}+O\left(\epsilon^{3}\right) .
$$

We next examine the second integral on the right-hand side of (5.2). Thus, by making use of (4.4), (6.2), (6.6), (A.13), (4.5) 2 and $\mathrm{v}=O(\epsilon)$, we obtain

$$
\begin{array}{r}
J \nu \mathbf{B}^{-1} \frac{\partial \mathbf{B}}{\partial \xi^{\alpha}}(\mathbf{v} \otimes \mathbf{v}) \mathbf{e}_{\alpha}=J_{0} \mathbf{F}^{-1} \frac{\partial \mathbf{F}}{\partial \xi^{\alpha}}\{(\nu \mathbf{v} \otimes \mathbf{v})-2 \Omega \bar{\nu}(\xi \mathbf{v} \otimes \mathbf{v})\} \mathbf{e}_{\alpha} \\
-J_{0} \bar{\nu} \mathbf{F}^{-1} \frac{\partial}{\partial \xi^{\alpha}}\left(\mathbf{F} \mathbf{W} \mathbf{F}^{-1}\right) \mathbf{F}(\xi \mathbf{v} \otimes \mathbf{v}) \mathbf{e}_{\alpha}+O\left(\epsilon^{2}\right), \\
\begin{array}{r}
J \mathbf{v} \mathbf{B}^{-1} \mathbf{F} \mathbf{W} \mathbf{v}=J_{0} \bar{\nu} \mathbf{W}(\mathrm{v} \mathbf{v})+O\left(\epsilon^{2}\right), \\
J \nu \Gamma(\mathbf{v}) \mathbf{B}^{-1} \mathbf{s}=-J_{0}\left\{\left[\mathbf{H} \cdot(\nu \mathbf{v} \otimes \mathbf{v})-2 \Omega \bar{\nu} \mathbf{H} \cdot(\xi \mathbf{v} \otimes \mathbf{v})-\bar{\nu} \mathbf{W}^{T} \mathbf{H} \cdot(\xi \mathbf{v} \otimes \mathbf{v})\right] \mathbf{I}\right. \\
+\bar{\nu}(\mathbf{H} \cdot(\xi \mathbf{v} \otimes \mathbf{v})) \mathbf{W}\} \mathbf{F}^{-1} \mathbf{s}+O\left(\epsilon^{2}\right) .
\end{array}
\end{array}
$$


We see then, by appeal to (6.1), (6.3), $a_{s} \equiv \mathbf{H} \overline{\mathbf{v}} \cdot \overline{\mathbf{v}}$, and $\tilde{a}_{s} \equiv \mathbf{H} \overline{\mathbf{v}} \cdot \mathbf{W} \overline{\mathbf{v}}$, that the estimation

$$
\begin{aligned}
\int_{0}^{h(\boldsymbol{\xi}, t)} & J \nu \boldsymbol{\Gamma}(\mathbf{v}, \mathrm{v}) d \xi=-J_{0} h \bar{\nu}\left(n_{2}^{s}-m_{3}^{s} \Omega h\right) \mathbf{F}^{-1} \frac{\partial \mathbf{F}}{\partial \xi^{\alpha}}(\overline{\mathbf{v}} \otimes \overline{\mathbf{v}}) \mathbf{e}_{\alpha} \\
& -J_{0} h \bar{\nu}\left\{\left[\left(n_{2}^{s}-m_{3}^{s} \Omega h\right) a_{s}-\frac{1}{2} m_{3}^{s} h \tilde{a}_{s}\right] \mathbf{I}+\frac{1}{2} m_{3}^{s} h a_{s} \mathbf{W}\right\} \mathbf{F}^{-1} \mathbf{s} \\
& +\frac{1}{2} J_{0} h^{2} m_{3}^{s} \bar{\nu} \mathbf{F}^{-1} \frac{\partial}{\partial \xi^{\alpha}}\left(\mathbf{F W} \mathbf{F}^{-1}\right) \mathbf{F}(\overline{\mathbf{v}} \otimes \overline{\mathbf{v}}) \mathbf{e}_{\alpha}+J_{0} h^{2} \beta_{s} \bar{\nu} \mathbf{W} \overline{\mathbf{v}}+O\left(\epsilon^{2+\gamma}\right)
\end{aligned}
$$

holds. In order to evaluate the last integral in (5.2), we first use the approximation

$$
c_{s}=c_{s}^{\star}+O(\epsilon), \quad c_{s}^{\star} \equiv \frac{c_{0} \bar{\nu}}{c_{0} \bar{\nu}+1-\bar{\nu}},
$$

and recall that $\tilde{p}_{s}=O(\epsilon), \tilde{p}_{f}=O(\epsilon)$, to show the relation

$$
J \stackrel{+}{\mathbf{m}}=J_{0}\left\{\left(1-c_{s}^{\star}\right) \tilde{p}_{s}^{\star}+\frac{c_{s}^{\star}}{c_{0}} \tilde{p}_{f}^{\star}\right\} \mathbf{M}_{0} \operatorname{Grad} \bar{\nu}+c_{D} J_{0}(1-2 \Omega \xi)(\mathbf{u}-\mathbf{v})+O\left(\epsilon^{1+\gamma}\right) .
$$

Then, replacing $\tilde{p}_{s}^{\star}, \tilde{p}_{f}^{\star}$ by their values as given in (A.6), we arrive at

$$
\begin{aligned}
\int_{0}^{h(\boldsymbol{\xi}, t)} J \stackrel{\mathbf{m}}{ } d \xi= & \frac{J_{0} h^{2}}{2\left(c_{0} \bar{\nu}+1-\bar{\nu}\right)}\left\{c+(1-\bar{\nu}) m_{3}^{s} a_{s}+\bar{\nu} m_{3}^{f} a_{f}\right\} \mathbf{M}_{0} \operatorname{Grad} \bar{\nu} \\
& +c_{D} J_{0} h\left\{\left(1-m_{1}^{f} \Omega h\right) \overline{\mathbf{u}}-\left(1-m_{1}^{s} \Omega h\right) \overline{\mathbf{v}}\right\}+O\left(\epsilon^{2+\gamma}\right) .
\end{aligned}
$$

Finally, substitution of (A.8) ${ }_{2}$, (A.10)-(A.12), (A.14)-(A.16) into (5.2) proves (7.7).

The governing equations (7.8), (7.9) describing the motion of the fluid constituent can be analogously deduced.

Acknowldgement We thank K. Hutter and F. Bouchut for valuable discussions and suggestions. We also acknowledge the anonymous referee for careful reading of the manuscript and suggestions; section 9 was added to the paper as proposed by the referee.

\section{REFERENCES}

[1] F. Bouchut and M. Westdickenberg, Gravity driven shallow water models for arbitrary topography, Commun. Math. Sci., 2(3), 359-389, 2004.

[2] F. Bouchut, E.D. Fernández-Nieto, A. Mangeney and P.-Y. Lagrée, On new erosion models of Savage-Hutter type for avalanches, Acta Mech., 199, 181-208, 2008.

[3] K.C. Chen and Y.C. Tai, Volume-weighted mixture theory for granular materials, Cont. Mech. Thermodyn., 19, 457-474, 2008.

[4] S. De Toni and P. Scotton, Two-dimensional mathematical and numerical model for the dynamics of granular avalanches, Cold Regions Science and Technology, 43, 36-48, 2005.

[5] S. Egashira, T. Itoh and H. Takeuchi, Transition mechanism of debris flows over rigid bed to over erodible bed, Phys. Chem. Earth (B), 26, 169-174, 2001.

[6] C.B. Harbitz, Snow Avalanche Modelling, Mapping and Warning in Europe (SAME), Report of the Fourth European Framework Programme: Environment and Climate, C.B. Harbitz (ed.), NGI, Norway 1998.

[7] K. Hutter, M. Siegel, S.B. Savage and Y. Nohguchi, Two-dimensional spreading of a granular avalanche down an inclined plane, Part I. Theory. Acta Mech., 100, 37-68, 1993.

[8] R.M. Iverson, The physics of debris flows, Rev. Geophys., 35, 245-296, 1997.

[9] R.M. Iverson and R.G. Denlinger, Flow of variably fluidized granular masses across threedimensional terrain. 1. Coulomb mixture theory, J. Geophysical Res., 106(B1), 537-552, 2001. 
[10] I. Luca, Y.C. Tai and C.Y. Kuo, Non-Cartesian topography based avalanche equations and approximations of gravity driven flows of ideal and viscous fluids, Math. Mod. Meth. Appl. Sci., 19, 127-171, 2009.

[11] I. Luca, K. Hutter, Y.C. Tai and C.Y. Kuo, A hierarchy of avalanche models on arbitrary topography, Acta Mech., to appear.

[12] M. Massoudi, Constitutive relations for the interaction force in multicomponent particulate flows, Int. J. Non-Linear Mech., 38, 313-336, 2003.

[13] S. Mambretti, E. Larcan and D. De Wrachien, Theoretical and experimental analysis of debris flow: rheology and two-phase modelling, Irrig. and Drain. DOI: 10.1002/ird.383, 2008.

[14] S. McDougall and O. Hungr, A model for the analysis of the rapid landslide motion across three dimensional terrain, Canad. Geotech. J., 41(6), 1084-1097, 2004.

[15] S. McDougall and O. Hungr, Dynamic modeling of entrainment in rapid landslides, Canad. Geotech. J., 42(5), 1437-1448, 2005.

[16] S.L. Passman, J.W. Nunziato and E.K. Walsh, A theory of multiphase mixtures, Rational Thermodynamics, C. Truesdell (ed.), Springer, New York, 1984.

[17] M. Pelanti, F. Bouchut and A. Mangeney, A Roe-type scheme for two-phase shallow granular flows over variable topography, ESAIM: M2AN 42, 851-885, 2008.

[18] E.B. Pitman and L. Le, A two-fluid model for avalanche and debris flows, Phil. Trans. R. Soc. A 363, 1573-1601, 2005.

[19] S.P. Pudasaini, Y. Wang and K. Hutter, Rapid motions of free surface avalanches down curved and twisted channels and their numerical simulation, Phil. Trans. R. Soc. A, 363, 15511571, 2005.

[20] S.P. Pudasaini, Y. Wang and K. Hutter, Modelling debris flows down general channels, Natural Hazards and Earth System Sciences, 5, 2005.

[21] S.P. Pudasaini, S.S. Hsiau, Y. Wang and K. Hutter, Velocity measurements in dry granular avalanches using particle image velocimetry technique and comparison with theoretical predictions, Physics Fluids, 17(9), 2005.

[22] S.P. Pudasaini and K. Hutter, Avalanche Dynamics, Berlin Heidelberg New York, Springer, 2007.

[23] J.A. Roberson and C.T. Crowe, Engineering Fluid Mechanics, Houghton-Miffin, Boston, 5th ed., 1993.

[24] S.B. Savage and K. Hutter, The motion of a finite mass of granular material down a rough incline, J. Fluid. Mech., 199, 177-215, 1989.

[25] L. Schneider and K. Hutter, Solid-fluid mixtures of frictional materials in geophysical and geotechnical context, to appear.

[26] Y.C. Tai and J.M.N.T. Gray, Limiting stress states in granular avalanches, Annals of Glaciology $26,272-276,1998$.

[27] Y.C. Tai, K. Hutter and J.M.N.T. Gray, Steady motion of a finite granular mass in a rotating drum, The Chinese Journal of Mechanics, 16, 67-72, 2000.

[28] Y.C. Tai and C.Y. Kuo, A new model of granular flows over general topography with erosion and deposition, Acta Mech., 199, 71-96, 2008.

[29] C. Truesdell and R. Toupin, Handbuch der Physik, III/1, ed. S. Flügge, Springer Verlag, Berlin, Göttingen, Heidelberg, 1960.

[30] C. Truesdell, Rational Thermodynamics, second edition, Mc Graw-Hill Series in Modern Applied Mathematics, New York 1969, Springer, New York, 1984. 